\title{
Quantum mechanical systems interacting with different polarizations of gravitational waves in noncommutative phase space
}

\author{
Anirban Saha* and Sunandan Gangopadhyay ${ }^{\dagger}$ \\ Department of Physics, West Bengal State University, \\ Barasat, North 24 Paraganas, West Bengal 700126, India \\ Swarup Saha \\ Department of Radiotherapy and Nuclear Medicine, \\ Barasat Cancer Research and Welfare Center, Barasat 700124, India \\ and Department of Physics, West Bengal State University, Barasat, \\ North 24 Paraganas, West Bengal 700124, India
}

\begin{abstract}
(Received 2 May 2016; revised manuscript received 28 February 2017; published 9 February 2018)
\end{abstract}
\begin{abstract}
Owing to the extreme smallness of any noncommutative scale that may exist in nature, both in the spatial and momentum sector of the quantum phase space, a credible possibility of their detection lies in the gravitational wave $(\mathrm{GW})$ detection scenario, where one effectively probes the relative length-scale variations $\sim \mathcal{O}\left[10^{-20}-10^{-23}\right]$. With this motivation, we have theoretically constructed how a free particle and a harmonic oscillator will respond to linearly and circularly polarized gravitational waves if their quantum mechanical phase space has a noncommutative structure. We critically analyze the formal solutions which show resonance behavior in the responses of both free particle and HO systems to GW with both kind of polarizations. We discuss the possible implications of these solutions in detecting noncommutativity in a GW detection experiment. We use the currently available upper-bound estimates on various noncommutative parameters to anticipate the relative importance of various terms in the solutions. We also argue how the quantum harmonic oscillator system we considered here can be very relevant in the context of the resonant bar detectors of GW which are already operational.
\end{abstract}

DOI: $10.1103 /$ PhysRevD.97.044015

\section{INTRODUCTION}

At the Planck scale, the spacetime is thought to have a granular structure much like the phase space of quantum mechanics $(\mathrm{QM})$. This is owing to the uncertainty induced in spatial coordinates due to sharp localization of events in space [1-3]. This granularity can be realized theoretically by describing the spacetime with a set of coordinates $x^{\mu}$, $(\mu=0,1,2,3)$ following a noncommutative (NC) algebra $\left[x^{\mu}, x^{\nu}\right]=i \theta^{\mu \nu}$, where $\theta^{\mu \nu}$ is a constant anti-symmetric tensor

\footnotetext{
*anirban@iucaa.ernet.in;

Visiting Associate in Inter University Centre for Astronomy and Astrophysics, Pune, India.

†sunandan.gangopadhyay@gmail.com; sunandan@iucaa.ernet.in;

Visiting Associate in Inter University Centre for Astronomy and Astrophysics (IUCAA), Pune, India.

¥saha18swarup@gmail.com

Published by the American Physical Society under the terms of the Creative Commons Attribution 4.0 International license. Further distribution of this work must maintain attribution to the author(s) and the published article's title, journal citation, and DOI. Funded by SCOAP ${ }^{3}$.
}

of second rank. This spacetime is referred to as canonical NC spacetime [4]. Theories defined on such spaces are generically called NC theories [5-7]. One can further extend the NC space[8] to a more general NC phase space [9-13] with both the canonical pairs following the algebra [14],

$$
\left[\hat{x}_{i}, \hat{p}_{j}\right]=i \tilde{h} \delta_{i j}, \quad\left[\hat{x}_{i}, \hat{x}_{j}\right]=i \theta \epsilon_{i j}, \quad\left[\hat{p}_{i}, \hat{p}_{j}\right]=i \bar{\theta} \epsilon_{i j}
$$

where $i, j=1,2 ; \tilde{h}=\hbar\left(1+\frac{\theta \bar{\theta}}{4 \hbar^{2}}\right)$ is the modified Planck's constant; $\theta$ and $\bar{\theta}$ are spatial and momentum NC parameters, respectively, and $\epsilon_{i j}=-\epsilon_{j i},\left(\epsilon_{12}=1\right)$ is the antisymmetric tensor in two dimensions. The main argument for considering a NC phase space is that noncommutativity between momenta arises naturally as a consequence of noncommutativity between coordinates, as momenta are defined as the partial derivatives of the action with respect to the coordinates [15]. Theories defined over a noncommutative phase space have also been furnished in [16-18] in context of the NC harmonic oscillator and NC Lorentz transformations.

The mathematical complexities aside, the biggest challenge in such NC theories is to identify experimentally 
detectable effects of noncommutativity owing to the extreme smallness of the NC parameters $\theta$ and $\bar{\theta}$ appearing in the algebra (1). Though such effects may only appear near the string/Planckian scale, it is hoped that some low energy relics may exist and their phenomenological consequences are currently being explored at the level of quantum mechanics [12,19-27]. Typical low energy nonaccelerator experiments are the Lamb-shift [28] and clockcomparison experiments [29], where the upper bound on the value of the canonical NC parameter was found to be $\theta \leq(10 \mathrm{TeV})^{-2}$ which corresponds to $4 \times 10^{-40} \mathrm{~m}^{2}$ for $\hbar=c=1$. On the other hand, such upper bounds on the momentum NC parameter [12,13] is $\bar{\theta} \leq 2.32 \times$ $10^{-61} \mathrm{~kg}^{2} \mathrm{~m}^{2} \mathrm{sec}^{-2}$ and time-space $\mathrm{NC}$ parameter $[23,24]$ is $\theta^{0 i} \leq 9.51 \times 10^{-18} \mathrm{~m}^{2}$ which are shown to be mutually consistent in [23]. These upper bounds correspond to the length-scale range $\sim 10^{-20} \mathrm{~m}$. Lookings at these bounds one cannot help but notice that at present the only potential possibility of finding the NC signature experimentally is in the high-precision gravitational wave (GW) detection experiments. Among the currently operating GW detectors (LIGO [30], VIRGO [31], GEO [32] and TAMA [33]) where interferometric techniques are being used, the LIGO detector has undergone new improvements and the Advanced LIGO [34] detectors has now reached a sensitivity goal where one can detect a length variation of the order of $\frac{\delta L}{L} \sim 10^{-23} / \sqrt{\mathrm{Hz}}$ or better. Indeed, LIGO has achieved the first-ever direct detection of a gravitational wave signal very recently [35], which was quickly followed by another one [36]. This has not only opened up a whole new channel for astronomical observations but also provided a means to directly look into the structure of spacetime at a length scale that has never been probed before. Various resonant detectors [37-42] are also striving to improve their sensitivity goals, specially with the new generation of spherical detectors like MiniGrail [43] and Schenberg [44]. Also, GW detectors based on matter-wave interferometry [45-47] have been suggested recently which claim similar strain sensitivity.

This roughly means that the currently existing and proposed GW detectors are effectively probing (or targeting to probe) a spacial length scale of order $10^{-20} \mathrm{~m}$ or beyond. These are, thus, ideally suited to monitor the fuzzyness introduced in distance measurements between test masses. If the space we live in indeed has a NC phase space structure in the quantum domain, the concept of distance is then fundamentally fuzzy. Thus even in the idealized situation where all classical and ordinary quantum noise sources are completely eliminated, the read-out of a GW detector would still pick up the effect of phase-space noncommutativity either as a noise source or something more interesting $[48,49]$.

Since NC phase-space structure is inherently quantum mechanical in nature, a quantum mechanical theory of the GW-matter interaction in an NC phase space would be necessary to predict the possible $\mathrm{NC}$ effects, noise sources or otherwise, in the GW detector read-outs. In this endeavor, we have recently adopted a systematic approach [50] to study the effect of linearly polarized GW(s) in the long-wavelength and low-velocity limit on the test matter, e.g., free particle and a harmonic oscillator (HO) $[51,52]$ in a space with $\mathrm{NC}$ coordinates. These studies show that while the spatial noncommutativity may not affect the response of a free particle to $\mathrm{GW}$ in a detectable manner, the response of a HO system to GW is significantly affected. Specifically, the spatial noncommutativity introduces a characteristic frequency into the $\mathrm{HO}$ system that shifts the frequency value where the $\mathrm{HO}$ will resonate with the GW. In [53], we have shown that when the noncommutative structure is generalized from the spatial sector to the whole phase space, even the response of the free particle to linearly polarized GW is significantly altered. The noncommutativity in the momentum sector brings in an oscillatory nature in the dynamical evolution of the free particle with a frequency characterized by the momentum NC parameter. Also in [54] we have shown that the free particle and $\mathrm{HO}$, in presence of only spatial noncommutativity, respond to a circularly polarized $\mathrm{GW}$ with the same basic features as they do to linearly polarized ones. Accounting for the circular polarization is nontrivial primarily because, unlike the linearly polarized case, here the polarization vectors evolve in time which makes the algorithm for computing the time evolution of the responding matter different from the former case.

In view of these results, one can immediately identify that three very pertinent questions emerge:

(1) Do the matter systems retain the basic similarities in their responses to both linear and circularly polarized GW (as has been found in the results of [54] mentioned above) when one generalizes from spatial noncommutativity to phase-space noncommutativity?

(2) Since the momentum sector of noncommutativity is known to introduce a novel frequency characterizing a fundamental momentum scale into the free particle system [53], making its time evolution periodic, how it will affect the HO system which already has a natural oscillation that can resonate with periodic $\mathrm{GW}$ signals?

(3) Are the effects of the spatial and momentum sector of the NC phase space distinguishable? Posed in a different form, if a NC signature is indeed detected in the response of free particle/HO to linearly/circularly polarized $\mathrm{GW}$, can one tell from which sector of the NC phase-space algebra did it originate?

We answer all these questions in the present paper by constructing a general quantum mechanical description in the NC phase space where both coordinate and momentum are assumed to follow noncommutative algebra (1), with both linear and circular polarization of GW interacting with free particle/ HO systems. We shall carry out the analysis for the linearly and circularly polarized GW signals 
separately since the algorithm to compute time evolution for the two cases are different. Owing to the extremely small size of both the spatial and momentum NC parameter, we shall restrict all the computations to first order in these $\mathrm{NC}$ parameters.

We must clarify at the outset that in the present paper we do not claim that our calculations readily apply to the current interferometric detectors, either ground-based or spacebased. In fact, the consideration for the response of a free particle to GW is only included to theoretically point out some interesting effects unique to the momentum noncommutativity which is otherwise absent (e.g., in the HO case). However the response of $\mathrm{GW}$ on the $\mathrm{HO}$ can be of much interest owing to its connection to the currently existing resonant detectors as we shall explain in due course.

This paper is organized as follows. In the next section, we describe the general methodology of our work. This involves obtaining a quantum mechanical description of a simple matter system interacting with GW in the NC phase space and computing its subsequent time-evolution. This will lay the theoretical foundation of the paper and also fix the notations. In Sec. III A, we compute the dynamics of a quantum particle which is otherwise free except for its coupling to circularly polarized $\mathrm{GW}$, in NC phase space. Analysis of this solution and comparison with our earlier work [53], where exclusively linearly polarized GWs was considered, will follow. We end this section discussing the importance of this result as well as the limitations on its applicability to existing GW detectors in Sec. III B. In Sec. IV, we present the quantum dynamics of a harmonic oscillator coupled to GW, again in the NC phase space, and analyze the results. Here interaction with both linearly as well as circularly polarized GW are worked out in IV A and IV B, respectively. We elaborate on how this computation can be relevant for the resonant bar-detectors of $\mathrm{GW}$ in Sec. IV C and briefly discuss the observational/detectional aspects and also the current status of resonant-detectors in IV D. We conclude in Sec. V.

\section{METHODOLOGY}

We start by noting that in the proper detector frame the geodesic deviation equation for a particle of mass $m$ subject to linearized GW takes [55] the form of a Newton's force equation. Specifically, in a potential $V(x)$ the equation is

$$
m \ddot{x}^{j}=-m R_{0, k 0}^{j} x^{k}-\partial_{j} V
$$

where dot denotes derivative with respect to the coordinate time of the proper detector frame[56], $x^{j}$ is the proper distance of the particle from the origin and $R^{j}{ }_{0, k 0}$ are the relevant components of the curvature tensor in terms of the metric perturbation $h_{\mu \nu}$ defined by[57]

$$
g_{\mu \nu}=\eta_{\mu \nu}+h_{\mu \nu} ; \quad\left|h_{\mu \nu}\right| \ll 1
$$

on the flat Minkowski background $\eta_{\mu \nu}$.

The gauge-choice $\left(h_{0 \mu}=0, h_{\mu \nu ;}{ }^{\mu}=0, h_{\mu}^{\mu}=0\right)$ that removes the unphysical degrees of freedom (DOF) and renders the GW transverse and traceless, has been made, so that only nontrivial components of the curvature tensor $R^{j}{ }_{0, k 0}=-\ddot{h}_{j k} / 2$ appear in Eq. (2). The two physical DOF brought out by this choice are referred as the $\times$ and + polarizations of GW and considering $z$ to be the propagation direction, the surviving components of the $2 \times 2$ matrix $h_{j k}$ in the transverse plane, $h_{11}=-h_{22}$ and $h_{12}=h_{21}$ represent these states, respectively.

Note that Eq. (2) can be used as long as the spatial velocities involved are nonrelativistic and $\left|x^{j}\right|$ is much smaller than the reduced wavelength $\frac{\lambda}{2 \pi}$ of GW. These conditions are collectively referred as the small-velocity and long-wavelength limit and met by resonant bar-detectors and earth bound interferometric detectors[58] with the origin of the coordinate system centered at the detector. This also ensures that in a plane-wave expansion of $\mathrm{GW}, h_{j k}=$ $\int\left(A_{j k} e^{i k x}+A_{j k}^{*} e^{-i k x}\right) d^{3} k /(2 \pi)^{3}$, the spatial part $e^{i \vec{k} \cdot \vec{x}} \approx 1$ all over the detector site. Thus our only concern is the timedependent part of the GW. If the polarization information contained in $A_{j k}$ is expressed in terms of the Pauli spin matrices, $h_{j k}$ takes the most convenient form,

$$
h_{j k}(t)=2 f\left(\varepsilon_{\times} \sigma_{j k}^{1}+\varepsilon_{+} \sigma_{j k}^{3}\right),
$$

where $2 f$ is the amplitude of the GW and $\left(\varepsilon_{\times}, \varepsilon_{+}\right)$are the two possible polarization states of the GW satisfying the condition $\varepsilon_{\times}^{2}+\varepsilon_{+}^{2}=1$ for all $t$. For linearly polarized GW, the frequency $\Omega$ is contained in the time-dependent amplitude $2 f(t)$ whereas for circularly polarized GW the timedependent polarization states $\left[\varepsilon_{\times}(t), \varepsilon_{+}(t)\right]$ contains the frequency $\Omega$.

\section{A. Constructing the quantum system interacting with gravitational waves in NC phase space}

In order to obtain a quantum mechanical description of the GW-matter interaction, we first obtain the appropriate classical Hamiltonian. This can be simply done by writing down the Lagrangian for the system (2), up to a total timederivative term as [50]

$$
\mathcal{L}=\frac{1}{2} m \dot{x}^{2}-m \Gamma^{j}{ }_{0 k} \dot{x}_{j} x^{k}-V(x,)
$$

where $R^{j}{ }_{0, k 0}=-\frac{d \Gamma_{0 k}^{j}}{d t}=-\ddot{h}_{j k} / 2$. Computing the canonical momentum $p_{j}=m \dot{x}_{j}-m \Gamma_{0 k}^{j} x^{k}$ corresponding to $x_{j}$, we write the Hamiltonian,

$$
H=\frac{1}{2 m}\left(p_{j}+m \Gamma_{0 k}^{j} x^{k}\right)^{2}+V(x) .
$$


Once we have the classical Hamiltonian we can have the NCQM description of the system simply by elevating the phase-space variables $\left(x^{j}, p_{j}\right)$ to operators $\left(\hat{x}^{j}, \hat{p}_{j}\right)$ and imposing the NC Heisenberg algebra (1). Note that this algebra can be mapped [22,28] to the standard $(\theta, \bar{\theta})=(0,0)$ Heisenberg algebra spanned by the operators $\hat{X}_{i}$ and $\hat{P}_{j}$ of the ordinary QM through the transformation equations

$$
\hat{x}_{i}=\hat{X}_{i}-\frac{1}{2 \hbar} \theta \epsilon_{i j} \hat{P}_{j}, \quad \hat{p}_{i}=\hat{P}_{i}+\frac{1}{2 \hbar} \bar{\theta} \epsilon_{i j} \hat{X}_{j} .
$$

Writing the NCQM version of Eq. (6) and employing the map (7), we obtain the effective Hamiltonian

$$
\begin{aligned}
\hat{H}= & \frac{\hat{P}_{j}^{2}}{2 m}+\Gamma_{0 k}^{j} \hat{X}_{j} \hat{P}_{k}+\frac{\bar{\theta}}{2 m \hbar} \epsilon_{j m} \hat{X}^{j} \hat{P}_{m}-\frac{\theta}{2 \hbar} \epsilon_{j m} \hat{P}_{m} \hat{P}_{k} \Gamma_{0 k}^{j} \\
& +\frac{\bar{\theta}}{2 \hbar} \epsilon_{j m} \hat{X}_{m} \hat{X}_{k} \Gamma_{0 k}^{j}+V\left(\hat{X}_{i}-\frac{1}{2 \hbar} \theta \epsilon_{i j} \hat{P}_{j}\right)
\end{aligned}
$$

which gives the equivalent description of the noncommutative system (6) in terms of the commutative operators $\hat{X}_{i}$ and $\hat{P}_{j}$. Since these operators admit the standard Heisenberg algebra, the rules of ordinary QM applies to (8). Also note that it has been demonstrated in various formulations of $\mathrm{NC}$ general relativity $[60,61]$ that any $\mathrm{NC}$ correction in the gravity sector is second order in the $\mathrm{NC}$ parameter. Therefore, in a first-order theory in NC phase space, the GW remains unaltered by any NC effects. Further note that the recent direct observations of $\mathrm{GW}$ signals of strain sensitivity $1.0 \times 10^{-21}-3.4 \times 10^{-22}$ reported in [35] clearly prove that up to a length scale of $10^{-25} \mathrm{~m}$ gravity behaves in a fairly classical way and, thus, we can safely assume that any possible NC correction, being inherently quantum in nature, would appear in the system through the matter part only.

\section{B. Tracking the time evolution}

To study the time evolution of the system, we introduce the raising and lowering operators:

$$
\begin{aligned}
& \hat{X}_{j}=\left(\frac{\hbar}{2 m \varpi}\right)^{1 / 2}\left(a_{j}+a_{j}^{\dagger}\right) \\
& \hat{P}_{j}=-i\left(\frac{\hbar m \varpi}{2}\right)^{1 / 2}\left(a_{j}-a_{j}^{\dagger}\right) .
\end{aligned}
$$

For the free particle case, $V(x)=0$, the frequency $\varpi$ is determined from the initial uncertainty in either the position or the momentum of the particle [50] whereas for the harmonic oscillator case $V(x)=\frac{1}{2} m \varpi^{2} x_{j}{ }^{2}, \varpi$ is identified with the natural frequency of the oscillator. Expressing the commutative equivalent effective Hamiltonian (8) in terms of $\left(a_{j}, a_{j}^{\dagger}\right)$ one can easily compute the time evolution of the system by

$$
\frac{d a_{j}(t)}{d t}=\frac{1}{i \hbar}\left[\hat{H}, a_{j}\right]
$$

using the algebra satisfied by the raising and lowering operators

$$
\left[a_{j}(t), a_{k}^{\dagger}(t)\right]=\delta_{j k} ; \quad\left[a_{j}(t), a_{k}(t)\right]=0=\left[a_{j}^{\dagger}(t), a_{k}^{\dagger}(t)\right]
$$

We further employ the time-dependent Bogoliubov transformation which relates the operators $a_{j}(t)$ and $a_{j}^{\dagger}(t)$ with their initial value at time $t=0$,

$$
\begin{aligned}
& a_{j}(t)=u_{j k}(t) a_{k}(0)+v_{j k}(t) a_{k}^{\dagger}(0) \\
& a_{j}^{\dagger}(t)=a_{k}^{\dagger}(0) \bar{u}_{k j}(t)+a_{k}(0) \bar{v}_{k j}(t),
\end{aligned}
$$

so that the time evolution of the system can be cast in terms of the generalized Bogoliubov coefficients $u_{j k}$ and $v_{j k}$ which are $2 \times 2$ complex matrices in the $x-y$ plane. Due to Eq. (11), they must satisfy

$$
u v^{T}=u^{T} v, \quad u u^{\dagger}-v v^{\dagger}=I,
$$

written in matrix form where $T$ denotes transpose, $\dagger$ denotes complex conjugate transpose and $I$ is the identity matrix. Since $a_{j}(t=0)=a_{j}(0)$, Eq. (12) imposes the boundary conditions

$$
u_{j k}(0)=I ; \quad v_{j k}(0)=0 .
$$

on $u_{j k}(t)$ and $v_{j k}(t)$. The final form of the equations of motion are written in terms of a pair of $2 \times 2$ matrices,

$$
\zeta_{j k}=u_{j k}-v_{j k}^{\dagger} ; \quad \xi_{j k}=u_{j k}+v_{j k}^{\dagger},
$$

which are in the $x-y$ plane.

\section{Case of linearly polarized $G W$}

The linearly polarized GW can be expressed in terms of the Pauli spin matrices [see Eq. (4)] as

$$
h_{j k}(t)=2 f(t)\left(\varepsilon_{\times} \sigma_{j k}^{1}+\varepsilon_{+} \sigma_{j k}^{3}\right),
$$

where $f(t)=f_{0} e^{i \Omega t}$ is the oscillating amplitude with constant frequency $\Omega$. To set a suitable boundary condition we shall assume that the GW hits the system at $t=0$. We can set the condition

$$
f_{0}=0, \quad \text { for } t \leq 0
$$

to this effect. Please note that though we have taken a periodic monochromatic GW signal in our calculation for simplicity, any realistic GW signal has continuously varying frequency. The detectors are blind to the GW signal away from their sensitive band and only starts to respond to it when the GW frequency is close to the sensitive band. Thus, Eq. (17) is a suitable boundary condition to impose that the system starts to respond to the GW at the start of the observation time $t=0$. The entire time-evolution that we shall compute is during this observation time only. The frequency of the $\mathrm{HO}$ system is assumed to be close to the GW frequency throughout this entire time. 
Since any $2 \times 2$ complex matrix can be written as a linear combination of the Pauli spin matrices and identity matrix, we also express the matrices $\zeta_{j k}$ and $\xi_{j k}$ as

$$
\begin{aligned}
& \zeta_{j k}(t)=A I_{j k}+B_{1} \sigma_{j k}^{1}+B_{2} \sigma_{j k}^{2}+B_{3} \sigma_{j k}^{3} \\
& \xi_{j k}(t)=C I_{j k}+D_{1} \sigma_{j k}^{1}+D_{2} \sigma_{j k}^{2}+D_{3} \sigma_{j k}^{3},
\end{aligned}
$$

so that the equations of motion will reduce to a set of coupled first-order differential equations in $A, B_{1}, B_{2}, B_{3}$, $C, D_{1}, D_{2}, D_{3}$. Noting that $|f(t)| \ll 1$, these equations can be solved iteratively about their $f(t)=0$ solution.

\section{Case of circularly polarized $G W$}

In case of circularly polarized $\mathrm{GW}$, the situation is slightly different. Here the $\mathrm{GW} h_{j k}$ can be written as

$$
h_{j k}(t)=2 f_{0}\left(\varepsilon_{1}(t) \sigma_{j k}^{1}+\varepsilon_{3}(t) \sigma_{j k}^{3}\right),
$$

where $2 f_{0}$ is the constant amplitude of the GW and the polarization vectors $\varepsilon_{1}(t)$ and $\varepsilon_{3}(t)$ evolve according to

$$
\frac{d \epsilon_{1}(t)}{d t}=-\Omega \epsilon_{3}(t), \quad \frac{d \epsilon_{3}(t)}{d t}=\Omega \epsilon_{1}(t),
$$

with $\Omega$ being a constant frequency. In this case, we proceed to solve the equations of motion as follows. First we remember that any $2 \times 2$ complex matrix $M$ can be written as a linear combination of the Pauli spin matrices and identity matrix as

$$
M=\theta_{0} I+\theta_{A} \sigma^{A},
$$

where $\theta_{0}$ and $\theta_{A}$ are complex numbers. Next, considering $\left(\theta_{A}\right), A=1,2,3$ as being a vector in a three-dimensional complex space. The polarization states of the GW can also be represented as a vector $\vec{\varepsilon}$ in this space. Considering $\vec{\varepsilon}, \dot{\vec{\varepsilon}}$ and $\vec{\varepsilon} \times \dot{\vec{\varepsilon}}$ are mutually orthogonal and, thus, form a natural directional triad, we can choose the trio as the coordinate axis for this space. Hence, we express the pair of matrices $(\chi, \xi)$ as

$$
\begin{aligned}
& \chi=A I+B \vec{\varepsilon} \cdot \vec{\sigma}+C \frac{\dot{\vec{\varepsilon}} \cdot \vec{\sigma}}{\Omega}+D i \frac{\vec{\varepsilon} \times \dot{\vec{\varepsilon}}}{\Omega} \cdot \vec{\sigma}, \\
& \xi=E I+F \vec{\varepsilon} \cdot \vec{\sigma}+G \frac{\dot{\vec{\varepsilon}} \cdot \vec{\sigma}}{\Omega}+H i \frac{\vec{\varepsilon} \times \dot{\vec{\varepsilon}}}{\Omega} \cdot \vec{\sigma},
\end{aligned}
$$

where $A, B, C, D, E, F, G, H$ can be complex functions. We, thus, reduce our equations of motion to a set of first-order differential equations for these complex functions which will be solved iteratively about the $f_{0}=0$ solution up to first order in the GW amplitude.

In the next two sections, we shall compute how the quantum mechanical free particle and the harmonic oscillator respond to different polarizations $\mathrm{GW}$ in an NC phase space and analyze the results and discuss there possible relevance/ limitations in context of the existing GW detectors.

\section{FREE PARTICLE INTERACTING WITH CIRCULARLY POLARIZED GW}

In this section, we first compute the quantum dynamics of a free particle interacting with circularly polarized GW assuming that the underlying phase space is noncommutative. We shall discuss the analytical features of the solutions. Then we move on to point out the importance of this model in detecting the possible existence of noncommutativity in momentum space. We also discuss the limitations of our model in context of the existing GW detection methods.

\section{A. Quantum dynamics of the system and time evolution}

We consider a quantum mechanical free particle interacting with circularly polarized GWs in NC phase space that obeys algebra (1). In this case, the potential is $V=0$ and in terms of the raising and lowering operators (9) the commutative equivalent of the NC phase-space Hamiltonian (8) takes the form

$$
\begin{aligned}
\hat{H}= & \frac{\hbar \varpi}{4}\left(2 a_{j}^{\dagger} a_{j}+1-a_{j}^{2}-a_{j}^{\dagger 2}\right)-\frac{i \hbar}{4} \dot{h}_{j k}\left(a_{j} a_{k}-a_{j}^{\dagger} a_{k}^{\dagger}\right) \\
& +\frac{i \bar{\theta}}{4 m} \epsilon_{j k} a_{j}^{\dagger} a_{k}+\frac{m \varpi \theta}{8} \epsilon_{j m} \dot{h}_{j k}\left(a_{m} a_{k}-a_{m} a_{k}^{\dagger}+\text { c.c. }\right) \\
& +\frac{\bar{\theta}}{8 m \varpi} \epsilon_{j m} \dot{h}_{j k}\left(a_{m} a_{k}+a_{m} a_{k}^{\dagger}+\text { c.c. }\right) .
\end{aligned}
$$

Hence, the Heisenberg equation of motion of $a_{j}(t)(10)$ reads

$$
\begin{aligned}
\frac{d a_{j}(t)}{d t}= & \frac{-i \varpi}{2}\left(a_{j}-a_{j}^{\dagger}\right)+\frac{1}{2} \dot{h}_{j k} a_{k}^{\dagger}+\frac{\bar{\theta}}{4 m \hbar} \epsilon_{j k} a_{k} \\
& +\frac{i m \varpi \theta}{8 \hbar}\left(\epsilon_{l j} \dot{h}_{l k}+\epsilon_{l k} \dot{h}_{l j}\right)\left(a_{k}-a_{k}^{\dagger}\right) \\
& -\frac{i \bar{\theta}}{8 m \varpi \hbar}\left(\epsilon_{l j} \dot{h}_{l k}+\epsilon_{l k} \dot{h}_{l j}\right)\left(a_{k}+a_{k}^{\dagger}\right)
\end{aligned}
$$

and that of $a_{j}^{\dagger}(t)$, given by the c.c. of the above equation. Using Eq. (12), the time evolution described in (26) can be cast in terms of the matrix pair $(\zeta, \xi)$ defined in (15):

$\dot{\zeta}_{j k}=-\frac{1}{2} \dot{h}_{j l} \zeta_{l k}+\frac{\bar{\theta}}{4 m \hbar} \epsilon_{j l} \zeta_{l k}-\frac{i \bar{\theta}}{4 m \varpi \hbar}\left(\epsilon_{l j} \dot{h}_{l p}+\epsilon_{l p} \dot{h}_{l j}\right) \xi_{p k}$

$$
\begin{aligned}
\dot{\xi}_{j k}= & -i \varpi \zeta_{j k}+\frac{1}{2} \dot{h}_{j l} \xi_{l k}+\frac{i m \varpi \theta}{4 \hbar}\left(\epsilon_{p l} \dot{h}_{j p}-\epsilon_{j p} \dot{h}_{p l}\right) \zeta_{l k} \\
& +\frac{\bar{\theta}}{4 m \hbar} \epsilon_{j l} \xi_{l k} .
\end{aligned}
$$

Since we want to consider interacting with circularly polarized GW, we substitute Eqs. (4), (21), (23), (24) in 
Eqs. (27), (28) and get the quantum dynamics of the system described in terms of a set of first-order differential equations,

$$
\begin{aligned}
& \dot{A}=-f_{0} \Omega C+\Lambda_{\bar{\theta}} D-\frac{i 4 \Lambda_{\bar{\theta}}}{\varpi} f_{0} \Omega F 0, \\
& \dot{B}=\Omega C+f_{0} \Omega D-\Lambda_{\bar{\theta}} C-\frac{i 4 \Lambda_{\bar{\theta}}}{\varpi} f_{0} \Omega E 0, \\
& \dot{C}=-\Omega B-f_{0} \Omega A+\Lambda_{\bar{\theta}} B-\frac{i 4 \Lambda_{\bar{\theta}}}{\varpi} f_{0} \Omega H, \\
& \dot{D}=f_{0} \Omega B-\Lambda_{\bar{\theta}} A-\frac{i 4 \Lambda_{\bar{\theta}}}{\varpi} f_{0} \Omega G, \\
& \dot{E}=-i \varpi A+f_{0} \Omega G+\Lambda_{\bar{\theta}} H+i \Lambda f_{0} \Omega B, \\
& \dot{F}=\Omega G-i \varpi B-f_{0} \Omega H-\Lambda_{\bar{\theta}} G+i \Lambda f_{0} \Omega A, \\
& \dot{G}=-\Omega F-i \varpi C+f_{0} \Omega E+\Lambda_{\bar{\theta}} F+i \Lambda f_{0} \Omega D, \\
& \dot{H}=-i \varpi D-f_{0} \Omega F-\Lambda_{\bar{\theta}} E+i \Lambda f_{0} \Omega C,
\end{aligned}
$$

to be solved to first order in the GW amplitude with boundary conditions, Eq. (14), which physically signifies that the GW hits the particle at $t=0$. Here,

$$
\Lambda_{\bar{\theta}}=\frac{\bar{\theta}}{4 m \hbar}
$$

is a new frequency that is characteristic of the $\mathrm{NC}$ momentum scale $\bar{\theta}$ and

$$
\Lambda=\frac{m \varpi \theta}{\hbar}
$$

is a dimensionless parameter with spatial NC parameter $\sqrt{\theta}$.

Solving Eq. (29), we get the solutions for the functions $A(t), B(t), C(t), D(t), E(t), F(t), G(t)$ and $H(t)$ as follows:

$$
\begin{aligned}
& A(t)=\cos \Lambda_{\bar{\theta}} t-f_{0} \Omega \frac{1}{\Omega-\Lambda_{\bar{\theta}}} N_{3}-4 \Lambda_{\bar{\theta}} f_{0} \Omega \frac{N_{2}}{\varpi\left(\Omega-\Lambda_{\bar{\theta}}\right)}-i \frac{4 \Lambda_{\bar{\theta}} f_{0} \Omega N_{1}}{\varpi\left(\Omega-\Lambda_{\bar{\theta}}\right)} \\
& B(t)=N_{1}-f_{0} \Omega \frac{\left(1-\cos \Lambda_{\bar{\theta}} t\right)}{\Lambda_{\bar{\theta}}}-4 f_{0} \Omega\left[t \sin \Lambda_{\bar{\theta}} t+\frac{1-\cos \Lambda_{\bar{\theta}} t}{\Lambda_{\bar{\theta}}} p o-i \frac{4 f_{0} \Omega \sin \Lambda_{\bar{\theta}} t}{\varpi}\right] \\
& C(t)=-N_{1}-\frac{f_{0} \Omega \sin \Lambda_{\bar{\theta}} t}{\Lambda_{\bar{\theta}}}-4 f_{0} \Omega\left[t \sin \Lambda_{\bar{\theta}} t+\frac{\left.1-\cos \Lambda_{\bar{\theta}} t\right]}{\Lambda_{\bar{\theta}}}\right]-i \frac{4 f_{0} \Omega \sin \Lambda_{\bar{\theta}} t}{\varpi} \\
& D(t)=-\sin \Lambda_{\bar{\theta}} t+f_{0} \Omega \frac{N_{1}}{\Omega-\Lambda_{\bar{\theta}}}-4 f_{0} \Omega \frac{\Lambda_{\bar{\theta}}}{\varpi\left(\Omega-\Lambda_{\bar{\theta}}\right)} N_{2}-i \frac{4 \Lambda_{\bar{\theta}} f_{0} \Omega N_{1}}{\varpi\left(\Omega-\Lambda_{\bar{\theta}}\right)} \\
& E(t)=\cos \Lambda_{\bar{\theta}} t+f_{0} \Omega \frac{N_{1}}{\Omega-\Lambda_{\bar{\theta}}}+i\left[-\frac{\varpi \sin \Lambda_{\bar{\theta}} t}{\Lambda_{\bar{\theta}}}+\frac{f_{0} \varpi}{\Omega-\Lambda_{\bar{\theta}}} N_{2}-\varpi\left(t \cos \Lambda_{\bar{\theta}} t+\frac{\sin \Lambda_{\bar{\theta}} t}{\Lambda_{\bar{\theta}}}\right)+\Lambda f_{0} \Omega \frac{N_{1}}{\Omega-\Lambda_{\bar{\theta}}}\right] \\
& F(t)=N_{1}-f_{0} \Omega \frac{\left(1-\cos \Lambda_{\bar{\theta}} t\right)}{\Lambda_{\bar{\theta}}}+i\left[N_{2}-\frac{\varpi N_{1}}{\Omega-\Lambda_{\bar{\theta}}}+\Lambda f_{0} \Omega \frac{\sin \Lambda_{\bar{\theta}} t}{\Lambda_{\bar{\theta}}}-f_{0} \Omega \varpi t \frac{\sin \Lambda_{\bar{\theta}} t}{\Lambda_{\bar{\theta}}}+f_{0} \Omega \varpi \frac{\left(1-\cos \Lambda_{\bar{\theta}} t\right)}{\left(\Lambda_{\bar{\theta}}\right)^{2}}\right] \\
& G(t)=-N_{1}+f_{0} \Omega \frac{\sin \Lambda_{\bar{\theta}} t}{\Lambda_{\bar{\theta}}}+i\left[N_{3}-\frac{\varpi N_{1}}{\Omega-\Lambda_{\bar{\theta}}}+\Lambda f_{0} \Omega \frac{1-\cos \Lambda_{\bar{\theta}} t}{\Lambda_{\bar{\theta}}}-\varpi t f_{0} \Omega \frac{\sin \Lambda_{\bar{\theta}} t}{\Lambda_{\bar{\theta}}}-f_{0} \Omega \varpi \frac{1-\cos \Lambda_{\bar{\theta}} t}{\Lambda_{\bar{\theta}}^{2}}\right] \\
& H(t)=-\sin \Lambda_{\bar{\theta}} t-\frac{f_{0} \Omega N_{1}}{\Omega-\Lambda_{\bar{\theta}}}+i\left[\frac{f_{0} \Omega}{\Omega-\Lambda_{\bar{\theta}}} N_{3}-\varpi t \sin \Lambda_{\bar{\theta}} t+\frac{\varpi\left(1-\cos \Lambda_{\bar{\theta}} t\right)}{\Lambda_{\bar{\theta}}}+\Lambda f_{0} \Omega \frac{N_{1}}{\Omega-\Lambda_{\bar{\theta}}}\right], a
\end{aligned}
$$

where $N_{1}, N_{2}, N_{3}$ are given by

$$
\begin{aligned}
& N_{1}=1-\cos \left(\Omega-\Lambda_{\bar{\theta}}\right) t \\
& N_{2}=\varpi\left[\frac{\sin \left(\Omega-\Lambda_{\bar{\theta}}\right) t+\cos \left(\Omega-\Lambda_{\bar{\theta}}\right) t-1}{\Omega-\Lambda_{\bar{\theta}}}-t\right] \\
& N_{3}=\varpi\left[\frac{\sin \left(\Omega-\Lambda_{\bar{\theta}}\right) t-\cos \left(\Omega-\Lambda_{\bar{\theta}}\right) t+1}{\Omega-\Lambda_{\bar{\theta}}}-t\right]
\end{aligned}
$$

Using these expressions in (23), (24), we can have the solution in terms of the matrix pair $(\zeta, \xi)$ which can be used in Eq. (15) to calculate the Bogoliubov coefficients. These are further substituted in (12) to obtain the timeevolution of the raising and lowering operators $a_{j}(t)$ and $a_{j}^{\dagger}(t)$ in terms of their initial values $a_{j}(0)$ and $a_{j}^{\dagger}(0)$. Using the definition of the raising and lowering operators (9) at initial time and any subsequent time yields the expectation value of the components of position and momentum of the particle at an arbitrary time $t$ in terms of their initial expectation values $\left[X_{1}(0), X_{2}(0)\right]$ and momentum $\left[P_{1}(0), P_{2}(0)\right]$. We give the explicit expression for $\left\langle X_{1}(t)\right\rangle$ and $\left\langle X_{2}(t)\right\rangle$ of the test bodies below. 


$$
\begin{aligned}
& \left\langle X_{1}(t)\right\rangle=\left[\cos \Lambda_{\bar{\theta}} t+\left(\epsilon_{3}-\epsilon_{1}\right) N_{1}\right] X_{1}(0)+\left[\sin \Lambda_{\bar{\theta}} t+\left(\epsilon_{1}+\epsilon_{3}\right) N_{1}\right] X_{2}(0)+\left[\varpi t \cos \Lambda_{\bar{\theta}} t+\left(N_{2} \epsilon_{3}+N_{3} \epsilon_{1}\right)\right. \\
& \left.+\frac{\varpi N_{1}}{\left(\Omega-\Lambda_{\bar{\theta}}\right)}\left(\epsilon_{1}+\epsilon_{3}\right)\right] \frac{P_{1}(0)}{m \varpi}+\left[\varpi\left\{\frac{\left(1-\cos \Lambda_{\bar{\theta}} t\right)}{\Lambda_{\bar{\theta}}}-t \sin \Lambda_{\bar{\theta}} t\right\}+\left(N_{2} \epsilon_{1}-N_{3} \epsilon_{3}\right)+\frac{\varpi N_{1}}{\left(\Omega-\Lambda_{\bar{\theta}}\right)}\left(\epsilon_{1}-\epsilon_{3}\right)\right] \frac{P_{2}(0)}{m \varpi} \\
& +f_{0} \Omega\left[-\frac{4 \Lambda_{\bar{\theta}} N_{1}}{\varpi\left(\Omega-\Lambda_{\bar{\theta}}\right)}-\frac{N_{3}}{\Omega-\Lambda_{\bar{\theta}}}-4\left(\epsilon_{1}-\epsilon_{3}\right)\left\{t \sin \Lambda_{\bar{\theta}} t-\frac{1-\cos \Lambda_{\bar{\theta}} t}{\Lambda_{\bar{\theta}}}\right\}-\left\{\frac{\epsilon_{3} \sin \Lambda_{\bar{\theta}} t-\epsilon_{1}\left(1-\cos \Lambda_{\bar{\theta}} t\right)}{\Lambda_{\bar{\theta}}}\right\}\right] X_{1}(0) \\
& +f_{0} \Omega\left[\frac{4 \Lambda_{\bar{\theta}} N_{2}}{\varpi\left(\Omega-\Lambda_{\bar{\theta}}\right)}-\frac{N_{1}}{\Omega-\Lambda_{\bar{\theta}}}-4\left(\epsilon_{1}+\epsilon_{3}\right)\left\{t \sin \Lambda_{\bar{\theta}} t-\frac{1-\cos \Lambda_{\bar{\theta}} t}{\Lambda_{\bar{\theta}}}\right\}-\left\{\frac{\epsilon_{1} \sin \Lambda_{\bar{\theta}} t+\epsilon_{3}\left(1-\cos \Lambda_{\bar{\theta}} t\right)}{\Lambda_{\bar{\theta}}}\right\}\right] X_{2}(0) \\
& +f_{0} \Omega\left[-\frac{N_{2}}{\Omega-\Lambda_{\bar{\theta}}}+\frac{4 \Lambda_{\bar{\theta}} N_{1}}{\varpi\left(\Omega-\Lambda_{\bar{\theta}}\right)}-\frac{\Lambda}{\Lambda_{\bar{\theta}}}\left\{\epsilon_{3} \sin \Lambda_{\bar{\theta}} t+\epsilon_{1}\left(1-\cos \Lambda_{\bar{\theta}} t\right)\right\}-\frac{\Lambda N_{1}}{\Omega-\Lambda_{\bar{\theta}}}-\frac{4 \sin \Lambda_{\bar{\theta}} t}{\varpi}\left(\epsilon_{3}+\epsilon_{1}\right)\right. \\
& \left.-\frac{\varpi}{\Lambda_{\bar{\theta}}}\left\{\frac{\left(1-\cos \Lambda_{\bar{\theta}} t\right)}{\Lambda_{\bar{\theta}}}-t \sin \Lambda_{\bar{\theta}} t\right\}\left(\epsilon_{3}+\epsilon_{1}\right)\right] \frac{P_{1}(0)}{m \varpi} \\
& +f_{0} \Omega\left[\frac{N_{3}}{\Omega-\Lambda_{\bar{\theta}}}-\frac{4 \Lambda_{\bar{\theta}} N_{1}}{\varpi\left(\Omega-\Lambda_{\bar{\theta}}\right)}-\frac{\Lambda}{\Lambda_{\bar{\theta}}}\left\{\epsilon_{1} \sin \Lambda_{\bar{\theta}} t-\epsilon_{3}\left(1-\cos \Lambda_{\bar{\theta}} t\right)\right\}+\frac{\Lambda N_{1}}{\Omega-\Lambda_{\bar{\theta}}}-\frac{4 \sin \Lambda_{\bar{\theta}} t}{\varpi}\left(\epsilon_{1}-\epsilon_{3}\right)\right. \\
& \left.-\frac{\varpi}{\Lambda_{\bar{\theta}}}\left\{\frac{\left(1-\cos \Lambda_{\bar{\theta}} t\right)}{\Lambda_{\bar{\theta}}}-t \sin \Lambda_{\bar{\theta}} t\right\}\left(\epsilon_{1}-\epsilon_{3}\right)\right] \frac{P_{2}(0)}{m \varpi} \\
& \left\langle X_{2}(t)\right\rangle=\left[\cos \Lambda_{\bar{\theta}} t-\left(\epsilon_{3}-\epsilon_{1}\right) N_{1}\right] X_{2}(0)+\left[\sin \Lambda_{\bar{\theta}} t+\left(\epsilon_{1}+\epsilon_{3}\right) N_{1}\right] X_{1}(0)+\left[\varpi t \cos \Lambda_{\bar{\theta}} t+\left(N_{2} \epsilon_{3}-N_{3} \epsilon_{1}\right)\right. \\
& \left.-\frac{\varpi N_{1}}{\left(\Omega-\Lambda_{\bar{\theta}}\right)}\left(\epsilon_{1}+\epsilon_{3}\right)\right] \frac{P_{2}(0)}{m \varpi}+\left[\varpi\left\{\frac{\left(1-\cos \Lambda_{\bar{\theta}} t\right)}{\Lambda_{\bar{\theta}}}-t \sin \Lambda_{\bar{\theta}} t\right\}+\left(N_{2} \epsilon_{1}-N_{3} \epsilon_{3}\right)+\frac{\varpi N_{1}}{\left(\Omega-\Lambda_{\bar{\theta}}\right)}\left(\epsilon_{1}-\epsilon_{3}\right)\right] \frac{P_{1}(0)}{m \varpi} \\
& +f_{0} \Omega\left[-\frac{4 \Lambda_{\bar{\theta}} N_{1}}{\varpi\left(\Omega-\Lambda_{\bar{\theta}}\right)}-\frac{N_{3}}{\Omega-\Lambda_{\bar{\theta}}}-4\left(\epsilon_{1}+\epsilon_{3}\right)\left\{t \sin \Lambda_{\bar{\theta}} t-\frac{1-\cos \Lambda_{\bar{\theta}} t}{\Lambda_{\bar{\theta}}}\right\}+\left\{\frac{\epsilon_{3} \sin \Lambda_{\bar{\theta}} t-\epsilon_{1}\left(1-\cos \Lambda_{\bar{\theta}} t\right)}{\Lambda_{\bar{\theta}}}\right\}\right] X_{2}(0) \\
& +f_{0} \Omega\left[\frac{4 \Lambda_{\bar{\theta}} N_{2}}{\varpi\left(\Omega-\Lambda_{\bar{\theta}}\right)}-\frac{N_{1}}{\Omega-\Lambda_{\bar{\theta}}}-4\left(\epsilon_{1}+\epsilon_{3}\right)\left\{t \sin \Lambda_{\bar{\theta}} t-\frac{1-\cos \Lambda_{\bar{\theta}} t}{\Lambda_{\bar{\theta}}}\right\}-\left\{\frac{\epsilon_{1} \sin \Lambda_{\bar{\theta}} t+\epsilon_{3}\left(1-\cos \Lambda_{\bar{\theta}} t\right)}{\Lambda_{\bar{\theta}}}\right\}\right] X_{1}(0) \\
& +f_{0} \Omega\left[-\frac{N_{2}}{\Omega-\Lambda_{\bar{\theta}}}+\frac{4 \Lambda_{\bar{\theta}} N_{1}}{\varpi\left(\Omega-\Lambda_{\bar{\theta}}\right)}-\frac{\Lambda}{\Lambda_{\bar{\theta}}}\left\{\epsilon_{3} \sin \Lambda_{\bar{\theta}} t+\epsilon_{1}\left(1-\cos \Lambda_{\bar{\theta}} t\right)\right\}-\frac{\Lambda N_{1}}{\Omega-\Lambda_{\bar{\theta}}}+\frac{4 \sin \Lambda_{\bar{\theta}} t}{\varpi}\left(\epsilon_{3}+\epsilon_{1}\right)\right. \\
& \left.+\frac{\varpi}{\Lambda_{\bar{\theta}}}\left\{\frac{\left(1-\cos \Lambda_{\bar{\theta}} t\right)}{\Lambda_{\bar{\theta}}}-t \sin \Lambda_{\bar{\theta}} t\right\}\left(\epsilon_{3}+\epsilon_{1}\right)\right] \frac{P_{2}(0)}{m \varpi} \\
& +f_{0} \Omega\left[\frac{N_{3}}{\Omega-\Lambda_{\bar{\theta}}}-\frac{4 \Lambda_{\bar{\theta}} N_{1}}{\varpi\left(\Omega-\Lambda_{\bar{\theta}}\right)}-\frac{\Lambda}{\Lambda_{\bar{\theta}}}\left\{\epsilon_{1} \sin \Lambda_{\bar{\theta}} t-\epsilon_{3}\left(1-\cos \Lambda_{\bar{\theta}} t\right)\right\}+\frac{\Lambda N_{1}}{\Omega-\Lambda_{\bar{\theta}}}+\frac{4 \sin \Lambda_{\bar{\theta}} t}{\varpi}\left(\epsilon_{1}-\epsilon_{3}\right)\right. \\
& \left.+\frac{\varpi}{\Lambda_{\bar{\theta}}}\left\{\frac{\left(1-\cos \Lambda_{\bar{\theta}} t\right)}{\Lambda_{\bar{\theta}}}-t \sin \Lambda_{\bar{\theta}} t\right\}\left(\epsilon_{1}-\epsilon_{3}\right)\right] \frac{P_{1}(0)}{m \varpi} .
\end{aligned}
$$

Note that the polarization vectors evolve according to Eq. (21) which has solutions

$$
\epsilon_{1}=\cos \Omega t, \quad \epsilon_{3}=\sin \Omega t .
$$

Before we study the salient features of the above result, let us point out that we have computed the expectation values for the coordinates $\left\langle X_{1}(t)\right\rangle$ and $\left\langle X_{2}(t)\right\rangle$ which are not the physical coordinates but correspond to a set of operators satisfying canonical commutation relations of the standard Heisenberg algebra of ordinary quantum mechanics. Hence, to present results for the physical coordinates and associated momenta; i.e., $\left(x_{i}, p_{i}\right)$ we can use Eq. (7). However, since we compute our results to first order in the NC parameters, this should not make a substantial difference to our result.

First, we look at the different terms of the above solutions. The first four square bracketed terms in both Eqs. (34), (35) show that the momentum noncommutativity makes the solution oscillatory with a characteristic frequency $\Lambda_{\bar{\theta}}$ even in the absence of any GW. The terms proportional to $N_{1}, N_{2}$ and $N_{3}$ appear there since we have chosen a directional triad which is rotating with a frequency $\Omega$. 
The rest of the terms are proportional to the $\mathrm{GW}$ amplitude $f_{0}$ and show the manifest effect of GW. In both Eqs. (34) and (35), among the terms with a $\left(\Omega-\Lambda_{\bar{\theta}}\right)^{-1}$ factor, some will grow rapidly near $\Omega=\Lambda_{\bar{\theta}}$ for nonzero $\bar{\theta}$. Considering the $\Omega \rightarrow \Lambda_{\bar{\theta}}$ limit for the following quantities,

$$
\begin{gathered}
\lim _{\Omega \rightarrow \Lambda_{\bar{\theta}}} \frac{N_{1}}{\Omega-\Lambda_{\bar{\theta}}}=0 \\
\lim _{\Omega \rightarrow \Lambda_{\bar{\theta}}} \frac{N_{2}}{\Omega-\Lambda_{\bar{\theta}}}=-\varpi\left(\frac{t^{2}}{2}+\frac{t}{\Omega-\Lambda_{\bar{\theta}}}\right) \\
\lim _{\Omega \rightarrow \Lambda_{\bar{\theta}}} \frac{N_{3}}{\Omega-\Lambda_{\bar{\theta}}}=\varpi\left(\frac{t^{2}}{2}-\frac{t}{\Omega-\Lambda_{\bar{\theta}}}\right),
\end{gathered}
$$

we see that in Eqs. (34), (35), only those terms with factors $\frac{N_{2}}{\Omega-\Lambda_{\bar{\theta}}}$ and $\frac{N_{3}}{\Omega-\Lambda_{\bar{\theta}}}$ will have this rapid growth effect. This we refer to as the "resonance behavior." Note that the resonance appears here typically between the oscillatory nature induced into the free particle motion by the noncommutativity among the momenta and the periodic GW. Thus it is a noncommutative effect. Also note that in the absence of GW there is no resonance effect. This can be verified by looking at the first four terms in both Eqs. (34) and (35) and noting that, though both $N_{2}$ and $N_{3}$ have the $\left(\Omega-\Lambda_{\bar{\theta}}\right)^{-1}$ factor, their limiting values are finite $\lim _{\Omega \rightarrow \Lambda_{\bar{\theta}}} N_{2}=\lim _{\Omega \rightarrow \Lambda_{\bar{\theta}}} N_{3}=\varpi t$.

Such noncommutativity-induced resonance behavior appeared earlier in [53] (where we considered response of a free particle to linearly polarized $\mathrm{GW}$ ) at the same value of frequency $\Lambda_{\bar{\theta}}=\frac{\bar{\theta}}{4 m \hbar}=\left(\frac{m}{m_{n}}\right)^{-1} 0.33 \mathrm{~Hz}$. This sub-Hz frequency range is estimated using the latest upperbound estimation of the momentum NC parameter [12] $\bar{\theta} \leq$ $2.32 \times 10^{-61} \mathrm{~kg}^{2} \mathrm{~m}^{2} \mathrm{sec}^{-2}$ and taking neutron mass $m_{n}=$ $167.32 \times 10^{-29} \mathrm{~kg}$ ) as the reference mass of a free particle. Reason for choosing neutron mass as reference is that we are considering the quantum dynamics of a free particle and only microscopic particles qualify. Thus we see from the present paper that the resonance point does not depend on the kind of polarization of the GW. This answers the first question posed in the introduction at least for the free particle case.

Another noticeable point in this connection is that the characteristic frequency $\Lambda_{\bar{\theta}}$, due to momentum noncommutativity, is ubiquitous in both Eqs. (34), (35), while the dimensionless parameter $\Lambda$, due to coordinate noncommutativity, only appears in a handful of terms, and such terms always carry the amplitude of the $\mathrm{GW} f_{0}$. Using the upperbound estimate[29] of the spatial NC parameter $\theta \approx$ $10^{-40} \mathrm{~m}^{2}$ and neutron mass as reference, this parameter value is estimated as $\Lambda=\frac{m \varpi \theta}{\hbar} \approx \varpi\left(\frac{m}{m_{n}}\right) 10^{-33}$. Since $\varpi$ is determined from the initial uncertainty of the momentum or position of the quantum particle, it cannot be large enough to make $\Lambda$ appreciable. Further, the smallness of GW strain amplitude $f_{0} \approx 10^{-23}$ suppresses such terms even further.
That spatial noncommutativity does not affect the response of a free particle to $\mathrm{GW}$ in a significant manner, a result established earlier in $[51,53]$ for linearly polarized GWs is, thus, shown to hold for circularly polarized GWs as well in the present paper, further answering the first question raised in the introduction.

A comment on the growth at resonance is in order now. The free particle, by its very definition comes without any damping term. However the transfer of energy from GW to the particle at resonance will not grow indefinitely since any realistic GW signal, which must have a varying frequency, will have a finite observation-time during which its frequency is close to the characteristic resonant frequency $\Lambda_{\bar{\theta}}$ of the system.

We finish this section by looking at the commutative limit of our solution. Since the NC corrections in the Hamiltonian [Eq. (40)] and the equations of motion [Eq. (26)] are all additive terms, our solution should have a smooth commutative limit $(\theta, \bar{\theta}) \rightarrow(0,0)$ i.e., $\left(\Lambda, \Lambda_{\bar{\theta}}\right) \rightarrow$ $(0,0)$ which it does have owing to factors like $\frac{\left(1-\cos \Lambda_{\bar{\theta}} t\right)}{\Lambda_{\bar{\theta}}}$ and $\frac{\sin \Lambda_{\bar{\theta}} t}{\Lambda_{\bar{\theta}}}$. The commutative limit of the solutions is explicitly given and certain consistency requirements of the general $\mathrm{NC}$ solution and its commutative limit is also discussed in appendix A 1.

\section{B. Significance vis-á-vis limited scope in the existing/planned detectors}

The above calculation suggests that the spatial NC effect enters the picture as a dimensionless parameter $\Lambda$ of ignorable size and therefore should go undetected whereas the momentum $\mathrm{NC}$ effect enters as a characteristic sub-HZ frequency $\Lambda_{\bar{\theta}}$ to induce a resonance with GW which is counter-intuitive for a free particle. The numerical value for this characteristic frequency $\Lambda_{\bar{\theta}} \sim 0.33 \mathrm{~Hz}$ mentioned above is estimated using the upper bound on the momentum NC parameter $\bar{\theta}$ and therefore it also serves as an upper bound only. The actual characteristic frequency may be smaller and push the resonance point further down to within the sensitivity window of a space-based detector. Note that we make reference to a space-borne detector here because of the sub-HZ upper bound on the characteristic resonant frequency since the sub- $\mathrm{Hz}$ window is plagued with various terrestrial noise sources in any ground-based GW detector. Thus an attempt to study this effect should be in a space-based scenario.

Unfortunately, the interaction model constructed in this section is not a very good description of the space-based interferometric GW detectors like eLISA [59] that is currently being planned. Though in eLISA[62] the freefloating test masses (gold-platinum cubes) are like free particles following the geodesic deviation equation in a proper-detector frame description [55], but our present result is not readily applicable to it, since the long-wavelength and low-velocity limit which we have used here, is not 
appropriate for eLISA. This is mainly because of the enormous arm-lengths of the interferometers in eLISA $(\sim 1$ million $\mathrm{KM})$ that makes the long-wavelength approximation invalid. Thus instead of the approximate form of the geodesic equation that we have used in Eq. (2), for eLISA, the full general relativistic equation of motion needs to be quantized to glean the NC phase-space effect. At any rate the present analysis points out the worthwhileness of such an attempt.

The long-wavelength-low-velocity limit is appropriate for a ground-based interferometric detectors like aLIGO where the physical arm-length of the interferometer is $\sim 4$ $\mathrm{KM}$ [63]. In the proper detector frame, the mirrors of such a ground-based detector does satisfy equations of motion that are in the form of a Newton's second law and, thus, our general scheme may be applicable to them in principle. However, whether a quantum mechanical description of a free particle applies to these mirrors, which is a macroscopic object, is a nontrivial issue that needs further investigation [64]. Besides, the characteristic frequency $\Lambda_{\bar{\theta}}$ depends inversely with the free particle's mass which, for a mirror would be much heavier than a neutron. Thus, at least at a first glance, the corresponding resonance point will be way below the sensitive bands of these groundbased interferometers which operate within a few hundred $\mathrm{Hz}-\mathrm{kHz}$ range.

A detector in space confined to smaller spatial region than eLISA and based on the principles of a quantummechanical free particle's response to GW will satisfy the long-wavelength and low-velocity limit. Since such detection scheme is not yet realized we can only point out, theoretically, the possible potential of such a scheme in identifying the existence of momentum noncommutativity. Therefore the free particle's response to GW and the effect of momentum noncommutativity on the response remains a purely theoretical consideration in the present paper. However, there are proposals for GW detectors of smaller spatial dimensions and sensitivity band in the lowfrequencies. For example, recently the combined atom and laser interferometry technique has been proposed that claims to have a sensitive band at lower frequency, namely 0.1-10 Hz. Also, the recently proposed Torsion-Bar Antenna (TOBA) $[65,66]$ for low-frequency GW observations may be relevant in this context.

In the next section, we shall consider how the HO system responds to GW in a $\mathrm{NC}$ phase space and discuss how it is a more relevant system as far as applicability to an existing GW detector is concerned.

\section{HARMONIC OSCILLATOR INTERACTING WITH LINEARLY AND CIRCULARLY POLARIZED GW}

We shall first work out the solutions of a $\mathrm{HO}$ reacting to linearly as well as circularly polarized GW assuming that the phase space has a NC structure. After analysing the features of the solutions we shall also discuss the relevance of this system in context of the resonant detectors [37-44] and their present status. For the $\mathrm{HO}$ case the potential energy is $V=\frac{1}{2} m \varpi^{2} x^{2}$ and the Hamiltonian, in terms of the raising and lowering operators, takes the form

$$
\begin{aligned}
\hat{H}= & \hbar \varpi\left(a_{j}^{\dagger} a_{j}+1\right)-\frac{i \hbar}{4} \dot{h}_{j k}\left(a_{j} a_{k}-a_{j}^{\dagger} a_{k}^{\dagger}\right) \\
& +\frac{m \varpi \theta}{8} \epsilon_{j m} \dot{h}_{j k}\left(a_{m} a_{k}-a_{m} a_{k}^{\dagger}+C . C\right) \\
& -\frac{i}{2} m \varpi^{2} \theta \epsilon_{j k} a_{j}^{\dagger} a_{k}+\frac{i \bar{\theta}}{4 m} \epsilon_{j k} a_{j}^{\dagger} a_{k} \\
& +\frac{\bar{\theta}}{8 m \varpi} \epsilon_{j m} \dot{h}_{j k}\left(a_{m} a_{k}+a_{m} a_{k}^{\dagger}+\text { C.C. }\right) .
\end{aligned}
$$

where C.C means complex conjugate as usual. Again working in the Heisenberg representation, the time evolution of $a_{j}(t)$ is given by

$$
\begin{aligned}
\frac{d a_{j}(t)}{d t}= & -i \varpi a^{j}+\frac{1}{2} \dot{h}_{j k} a_{k}^{\dagger}-\frac{m \varpi^{2} \theta}{2 \hbar} \epsilon_{j k} a_{k}+\frac{i m \varpi \theta}{8 \hbar} \\
& \times\left(\epsilon_{l j} \dot{h}_{l k}+\epsilon_{l k} \dot{h}_{l j}\right)\left(a_{k}-a_{k}^{\dagger}\right)+\frac{\bar{\theta}}{4 m \hbar} \epsilon_{j k} a_{k} \\
& -\frac{i \bar{\theta}}{8 m \varpi \hbar}\left(\epsilon_{l j} \dot{h}_{l k}+\epsilon_{l k} \dot{h}_{l j}\right)\left(a_{k}+a_{k}^{\dagger}\right)
\end{aligned}
$$

and that of $a_{j}^{\dagger}(t)$ is the c.c. of the above equation. In terms of the matrix pair $(\zeta, \xi)$ in (15), the time-evolution equations take the form

$$
\begin{aligned}
\dot{\zeta}_{j k}= & -i \varpi \xi_{j k}-\frac{1}{2} \dot{h}_{j l} \zeta_{l k}-\frac{m \varpi^{2} \theta}{2 \hbar} \epsilon_{j l} \zeta_{l k}+\frac{\bar{\theta}}{4 m \hbar} \epsilon_{j l} \zeta_{l k} \\
& -\frac{i \bar{\theta}}{4 m \varpi \hbar}\left(\epsilon_{l j} \dot{h}_{l p}+\epsilon_{l p} \dot{h}_{l j}\right) \xi_{p k} \\
\dot{\xi}_{j k}= & -i \varpi \zeta_{j k}+\frac{1}{2} \dot{h}_{j l} \xi_{l k}+\Theta_{j l} \zeta_{l k}-\frac{m \varpi^{2} \theta}{2 \hbar} \epsilon_{j l} \xi_{l k} \\
& +\frac{\bar{\theta}}{4 m \hbar} \epsilon_{j l} \xi_{l k},
\end{aligned}
$$

where $\Theta_{j l}$ is the term reflecting the interplay of noncommutativity with GW

$$
\Theta_{j l}=\frac{i m \varpi \theta}{4 \hbar}\left(\dot{h}_{j m} \epsilon_{m l}-\epsilon_{j m} \dot{h}_{m l}\right) .
$$

In the following, we shall solve Eqs. (42) (43) for the special cases of linearly and circularly polarized GW, respectively.

\section{A. Time evolution under linearly polarized GW}

Referring to (16) and (18), (19), we substitute for $h_{i j}$ and $(\zeta, \xi)$ in Eqs. (42), (43) and comparing the coefficients of $I$ and $\sigma$ matrices, we get a set of first-order differential equations for $A, B_{1}, B_{2}, B_{3}, C, D_{1}, D_{2}, D_{3}$ : 


$$
\begin{aligned}
\dot{A}= & -i \varpi C-\dot{f}\left(\varepsilon_{1} B_{1}+\varepsilon_{3} B_{3}\right)-4 i \frac{\Lambda_{\bar{\theta}}}{\varpi} \dot{f}\left(\varepsilon_{3} D_{1}-\varepsilon_{1} D_{3}\right) \\
& -i \Lambda_{0} B_{2} \\
\dot{B}_{1}= & -i \varpi D_{1}-\dot{f}\left(\varepsilon_{1} A-i \varepsilon_{3} B_{2}\right)-4 \frac{\Lambda_{\bar{\theta}}}{\varpi} \dot{f}\left(i \varepsilon_{3} C-\varepsilon_{1} D_{2}\right) \\
& +\Lambda_{0} B_{3} \\
\dot{B}_{2}= & -i \varpi D_{2}-i \dot{f}\left(\varepsilon_{3} B_{1}-\varepsilon_{1} B_{3}\right)-4 \frac{\Lambda_{\bar{\theta}}}{\varpi} \dot{f}\left(\varepsilon_{1} D_{1}+\varepsilon_{3} D_{3}\right) \\
& -i \Lambda_{0} A \\
\dot{B}_{3}= & -i \varpi D_{3}-\dot{f}\left(\varepsilon_{3} A+i \varepsilon_{1} B_{2}\right)+4 \frac{\Lambda_{\bar{\theta}}}{\varpi} \dot{f}\left(i \varepsilon_{1} C+\varepsilon_{3} D_{2}\right) \\
& -\Lambda_{0} B_{1} \\
\dot{C}_{=} & -i \varpi A+\dot{f}\left(\varepsilon_{1} D_{1}+\varepsilon_{3} D_{3}\right)+i \Lambda \dot{f}\left(\varepsilon_{3} B_{1}-\varepsilon_{1} B_{3}\right) \\
& -i \Lambda_{0} D_{2} \\
\dot{D}_{1}= & -i \varpi B_{1}+\dot{f}\left(\varepsilon_{1} C-i \varepsilon_{3} D_{2}\right)+\Lambda \dot{f}\left(i \varepsilon_{3} A-\varepsilon_{1} B_{2}\right) \\
& +\Lambda_{0} D_{3} \\
\dot{D}_{2}= & -i \varpi B_{2}+i \dot{f}\left(\varepsilon_{3} D_{1}-\varepsilon_{1} D_{3}\right)+\Lambda \dot{f}\left(\varepsilon_{1} B_{1}+\varepsilon_{3} B_{3}\right) \\
& -i \Lambda_{0} C \\
\dot{D}_{3}= & -i \varpi B_{3}+\dot{f}\left(\varepsilon_{3} C+i \varepsilon_{1} D_{2}\right)-\Lambda \dot{f}\left(i \varepsilon_{1} A+\varepsilon_{3} B_{2}\right) \\
& -\Lambda_{0} D_{1} .
\end{aligned}
$$

Here, the dot represents derivative with respect to time $t . \Lambda$ is the same dimensionless parameter carrying the spatial NC scale defined in (31) and

$$
\Lambda_{0}=\Lambda_{\theta}-\Lambda_{\bar{\theta}}
$$

is the difference between the frequency characterized by the spatial NC scale $\theta$ given by

$$
\Lambda_{\theta}=\frac{m \varpi^{2} \theta}{2 \hbar}
$$

and the frequency $\Lambda_{\bar{\theta}}$ characterized by the momentum NC scale $\bar{\theta}$ defined earlier in (30). Once we arrive at the solutions to the present problem, we shall provide estimates of these characteristic frequencies in context of the physical systems where the quantum harmonic oscillators we are discussing can be realized.

Noting that the GW strain amplitude is very small $|f(t)| \ll 1$, we again solve the above set of equations iteratively about its $f(t)=0$ solution. The appropriate boundary conditions to apply are again (14), (17). We obtain the following solutions to first order in the gravitational wave amplitude. We also restrict ourselves to first order in the NC parameter:

$$
\begin{aligned}
A(t) & =C(t)=e^{-i \varpi t} \cos \left(\Lambda_{0} t\right) \\
B_{2}(t) & =D_{2}(t)=-i e^{-i \varpi t} \sin \left(\Lambda_{0} t\right) \\
B_{1}(t) & =\left(\epsilon_{1}+i \frac{4 \Lambda_{\bar{\theta}} \epsilon_{3}}{\varpi}\right) K_{1}+\left(\epsilon_{3}+i \frac{4 \Lambda_{\bar{\theta}} \epsilon_{1}}{\varpi}\right) K_{2} \\
B_{3}(t) & =\left(\epsilon_{3}+i \frac{4 \Lambda_{\bar{\theta}} \epsilon_{1}}{\varpi}\right) K_{1}-\left(\epsilon_{1}+i \frac{4 \Lambda_{\bar{\theta}} \epsilon_{3}}{\varpi}\right) K_{2} \\
D_{1}(t) & =-\left(\epsilon_{1}+\frac{i \Lambda \epsilon_{3}}{4}\right) K_{1}-\left(\epsilon_{3}+\frac{i \Lambda \epsilon_{1}}{4}\right) K_{2} \\
D_{3}(t) & =-\left(\epsilon_{3}+\frac{i \Lambda \epsilon_{1}}{4}\right) K_{1}+\left(\epsilon_{1}+\frac{i \Lambda \epsilon_{3}}{4}\right) K_{2}
\end{aligned}
$$

with

$$
\begin{aligned}
K_{1}= & -e^{-i \varpi t} \cos \left(\Lambda_{0} t\right) f(t)+\varpi^{2} \int_{0}^{t} h_{1}\left(t^{\prime}\right) d t^{\prime} \\
& -2 i \varpi \int_{0}^{t} d t^{\prime} e^{i \varpi\left(t-t^{\prime}\right)} \cos \left(\Lambda_{0} t^{\prime}\right) f\left(t^{\prime}\right) \\
K_{2}= & e^{-i \varpi t} \sin \left(\Lambda_{0} t\right) f(t)-\varpi^{2} \int_{0}^{t} h_{2}\left(t^{\prime}\right) d t^{\prime} \\
& +2 i \varpi \int_{0}^{t} d t^{\prime} e^{i \varpi\left(t-t^{\prime}\right)} \sin \left(\Lambda_{0} t^{\prime}\right) f\left(t^{\prime}\right)
\end{aligned}
$$

and

$$
\begin{aligned}
& h_{1}(t)=\int_{0}^{t} d t^{\prime} e^{-i \varpi t^{\prime}} \cos \left(\Lambda_{0} t^{\prime}\right) f\left(t^{\prime}\right) \\
& h_{2}(t)=\int_{0}^{t} d t^{\prime} e^{-i \varpi t^{\prime}} \sin \left(\Lambda_{0} t^{\prime}\right) f\left(t^{\prime}\right) .
\end{aligned}
$$

To work out the above integrals, one needs specific GW wave form $f(t)$. In the present paper, we shall take a monochromatic sinusoidal wave form of frequency $\Omega$,

$$
f(t)=f_{0} e^{i \Omega t} .
$$

Once we have the solutions (48), the system is essentially solved and all that remains is to compute all the way back to the expectation value of the components of position and momentum of the particle at an arbitrary time $t$ in terms of their initial position $\left[X_{1}(0), X_{2}(0)\right]$ and momentum $\left[P_{1}(0), P_{2}(0)\right]$ expectation values. How that can be done has been discussed in Sec. III A [below (33)]. The explicit solutions for $\left\langle X_{1}(t)\right\rangle$ and $\left\langle X_{2}(t)\right\rangle$ are given in terms of the dimensionless functions

$$
\begin{aligned}
q_{1}(t)= & -\frac{1}{2}\left(\cos \Delta \varpi_{+} t+\cos \Delta \varpi_{-} t\right) \\
& +\frac{\varpi}{\Delta \varpi^{2}-\Lambda_{0}^{2}}\left[-k_{1}(t) \cos \varpi t+k_{2}(t) \sin \varpi t\right] \\
& +\frac{\varpi^{2}}{2}\left[-\frac{\sin \Delta \varpi_{-} t}{\left(\Delta \varpi_{-}\right)^{2}}+\frac{\sin \Delta \varpi_{+} t}{\left(\Delta \varpi_{+}\right)^{2}}+\frac{4 \Delta \varpi \Lambda_{0}}{\left(\Delta \varpi^{2}-\Lambda_{0}^{2}\right)^{2}}\right]
\end{aligned}
$$


$q_{2}(t)=-\frac{1}{2}\left(\sin \Delta \varpi_{+} t+\sin \Delta \varpi_{-} t\right)-\frac{\varpi^{2}}{\Delta \varpi^{2}-\Lambda_{0}^{2}}\left[k_{1}(t) \sin \varpi t+k_{2}(t) \cos \varpi t\right]+\frac{\varpi^{2}}{2}\left[\frac{\cos \Delta \varpi_{-} t}{\left(\Delta \varpi_{-}\right)^{2}}-\frac{\cos \Delta \varpi_{+} t}{\left(\Delta \varpi_{+}\right)^{2}}-\frac{2 \Lambda_{0} t}{\left(\Delta \varpi^{2}-\Lambda_{0}^{2}\right)}\right]$

$q_{3}(t)=\frac{1}{2}\left(\sin \Delta \varpi_{+} t-\sin \Delta \varpi_{-} t\right)-\frac{\varpi}{\Delta \varpi^{2}-\Lambda_{0}^{2}}\left[k_{1}(t) \sin \varpi t-k_{2}(t) \cos \varpi t\right]+\frac{\varpi^{2}}{2}\left[\frac{\sin \Delta \varpi_{-} t}{\left(\Delta \varpi_{-}\right)^{2}}-\frac{\sin \Delta \varpi_{+} t}{\left(\Delta \varpi_{+}\right)^{2}}+\frac{2\left(\Delta \varpi^{2}+\Lambda_{0}^{2}\right)}{\left(\Delta \varpi^{2}-\Lambda_{0}^{2}\right)^{2}}\right]$

$q_{4}(t)=-\frac{1}{2}\left(\cos \Delta \varpi_{+} t-\cos \Delta \varpi_{-} t\right)-\frac{\varpi}{\Delta \varpi^{2}-\Lambda_{0}^{2}}\left[k_{1}(t) \cos \varpi t+k_{2}(t) \sin \varpi t\right]-\frac{\varpi^{2}}{2}\left[\frac{\cos \Delta \varpi_{-} t}{\left(\Delta \varpi_{-}\right)^{2}}+\frac{\cos \Delta \varpi_{+} t}{\left(\Delta \varpi_{+}\right)^{2}}-\frac{2 \Delta \varpi^{2}}{\left(\Delta \varpi^{2}-\Lambda_{0}^{2}\right)}\right]$

and functions with the dimension of frequency

$$
\begin{gathered}
k_{1}(t)=2 \Delta \varpi-\Omega_{-} \cos \left(\Omega_{+} t\right)+\Omega_{+} \cos \left(\Omega_{-} t\right) \\
k_{2}(t)=\Omega_{+} \sin \left(\Omega_{-} t\right)+\Omega_{-} \sin \left(\Omega_{+} t\right)
\end{gathered}
$$

as the following:

$$
\begin{aligned}
\left\langle X_{1}(t)\right\rangle= & \frac{\left(\cos \varpi_{-} t+\cos \varpi_{+} t\right)}{2} X_{1}(0)+\frac{\left(\sin \varpi_{-} t+\sin \varpi_{+} t\right)}{2 m \varpi} P_{1}(0)+\frac{\left(\cos \varpi_{+} t-\cos \varpi_{-} t\right)}{2} X_{2}(0)+\frac{\left(\sin \varpi_{+} t-\sin \varpi_{-} t\right)}{2 m \varpi} P_{2}(0) \\
& -f_{0}\left[q_{1}(t)-\frac{\Lambda}{4} q_{4}(t)\right]\left[\epsilon_{3} X_{1}(0)+\epsilon_{1} X_{2}(0)\right]+f_{0}\left[q_{3}(t)+\frac{\Lambda}{4} q_{2}(t)\right]\left[\epsilon_{1} X_{1}(0)+\epsilon_{3} X_{2}(0)\right] \\
& +f_{0}\left[-q_{2}(t)+\frac{4 \Lambda_{\bar{\theta}}}{\varpi} q_{3}(t)\right]\left[\frac{\epsilon_{3} P_{1}(0)+\epsilon_{1} P_{2}(0)}{m \varpi}\right]-f_{0}\left[q_{4}(t)+\frac{4 \Lambda_{\bar{\theta}}}{\varpi} q_{1}(t)\right]\left[\frac{\epsilon_{1} P_{1}(0)-\epsilon_{3} P_{2}(0)}{m \varpi}\right] \\
\left\langle X_{2}(t)\right\rangle= & \frac{\left(\cos \varpi_{-} t+\cos \varpi_{+} t\right)}{2} X_{2}(0)+\frac{\left(\sin \varpi_{-} t+\sin \varpi_{+} t\right)}{2 m \varpi} P_{2}(0)-\frac{\left(\cos \varpi_{+} t-\cos \varpi_{-} t\right)}{2} X_{1}(0)-\frac{\left(\sin \varpi_{+} t-\sin \varpi_{-} t\right)}{2 m \varpi} P_{1}(0) \\
& +f_{0}\left[q_{1}(t)-\frac{\Lambda}{4} q_{4}(t)\right]\left[\epsilon_{3} X_{2}(0)-\epsilon_{1} X_{1}(0)\right]+f_{0}\left[q_{3}(t)+\frac{\Lambda}{4} q_{2}(t)\right]\left[-\epsilon_{1} X_{2}(0)+\epsilon_{3} X_{1}(0)\right] \\
& +f_{0}\left[-q_{2}(t)+\frac{4 \Lambda_{\bar{\theta}}}{\varpi} q_{3}(t)\right]\left[\frac{-\epsilon_{3} P_{2}(0)+\epsilon_{1} P_{1}(0)}{m \varpi}\right]-f_{0}\left[q_{4}(t)+\frac{4 \Lambda_{\bar{\theta}}}{\varpi} q_{1}(t)\right]\left[\frac{-\epsilon_{1} P_{2}(0)-\epsilon_{3} P_{1}(0)}{m \varpi}\right] .
\end{aligned}
$$

Note that, in the solution, various combinations of the frequencies present in the system appear. We list them in the following for ready reference. Take special note of the fact that the two frequencies $\Lambda_{\theta}$ and $\Lambda_{\bar{\theta}}$, characterizing, respectively, the noncommutativity of the spatial and the momentum sector, only appear as a combination $\Lambda_{0}$, defined in (46) and hereafter referred to as the characteristic frequency of the NC phase space, in all sinusoidal terms in the solutions (58) and (59).

$$
\begin{gathered}
\Delta \varpi=\varpi-\Omega(\text { difference of the frequencies of the } \mathrm{HO} \text { and that of the GW signal }) \\
\varpi_{ \pm}=\varpi \pm \Lambda_{0}\left(\text { natural frequency of the } \mathrm{HO}, \text { shifted by } \Lambda_{0}\right) \\
\Omega_{ \pm}=\Omega \pm \Lambda_{0}\left(\text { frequency of the } \mathrm{GW}, \text { shifted by } \Lambda_{0}\right) \\
\Delta \varpi_{ \pm}=\Delta \varpi \pm \Lambda_{0}\left(\text { difference of the frequencies of the HO and } \mathrm{GW}, \text { shifted by } \Lambda_{0}\right) .
\end{gathered}
$$

As in the free particle case, we start by looking at the different terms in the solutions critically. The first four terms in both (58), (59) are independent of any GW effect and only show the standard HO oscillator solution with the natural frequency $\varpi$ shifted by the characteristic frequency coming from NC phase space. The remaining terms are the combined effect of the GW and the NC phase space. Owing to the smallness of the GW strain amplitude $f_{0}$, these terms 
are ordinarily small. However, owing to the presence of factors $\Delta \varpi_{ \pm}$in the denominator, when

$$
\Delta \varpi_{ \pm}=0
$$

some terms in the dimensionless functions in (52), (53), (54), (55) will grow, thus amplifying the effect of the GW on the system. Here, also, we refer to this phenomenon as resonance, like in the free particle case in Sec. III A.

Equation (64) is very interesting due to the following reason. Later in this section we shall argue that the quantum $\mathrm{HO}$ considered here represents the phonon modes excited by the GW in a resonant detector. Ordinarily the resonant bar is expected to have only one sensitive frequency $\varpi$, uniquely determined by the size and material of the bar, where the resonance would occur, if there is no $\mathrm{NC}$ effect. However, incorporating the NC phase-space effect we see from Eq. (64) that both spatial and momentum NC parameters will enter the picture and combine to form a characteristic frequency of the NC phase space $\Lambda_{0}=\Lambda_{\theta}-\Lambda_{\bar{\theta}}$ and make the bar (with its one intrinsic frequency $\varpi$ ) resonate with GW signals at two different frequencies:

$$
\begin{aligned}
& \Omega=\varpi+\left(\Lambda_{\theta}-\Lambda_{\bar{\theta}}\right) \\
& \Omega=\varpi-\left(\Lambda_{\theta}-\Lambda_{\bar{\theta}}\right)
\end{aligned}
$$

Any realistic GW signal will have a time-varying frequency and in that case there is a possibility, however small, that the variation of the incoming GW signal frequency would cover both the resonance points and then the bar will resonate at these frequencies in a single detection event. However, note that for our purpose, i.e., to have evidence of an NC effect, the bar need not resonate at both the frequencies for the same GW signal in a single detection event. The same bar detector resonating to two different GW signals at two evenly placed frequencies, centering the intrinsic frequency of the bar, in two separate detection events should be good enough as evidence of noncommutativity.

To elaborate, if the intrinsic frequency of a monochromatic source of GW is $\Omega$ (say), then the resonance must occur at $\Omega$ and can be achieved/observed only if we have a bar detector operating at that frequency. Thus whichever frequency we observe the resonance at is the frequency of the monochromatic GW source. As we shall discuss in details later, the intrinsic frequency $\varpi$ of the fundamental mode of a bar detector of GW is known[55] from the sound speed in the bar material $v_{s}$ and length of the bar $L$, through the relation $\varpi=\frac{\pi v_{s}}{L}$. Therefore, if the resonance is observed to occur at $\varpi$ itself, (i.e., $\Omega=\varpi$ ) then we must conclude that there is no noncommutativity in space. On the other hand, if the resonance is observed to occur at a frequency different from the $\varpi$ value, (i.e., at $\Omega \neq \varpi$ ) then that difference $(|\Omega-\varpi|)$, as per our calculation, is due to noncommutativity of space and equals the characteristic
NC frequency parameter $\Lambda_{0}$. Note that even if the GW source is monochromatic (say $\Omega$ ) and resonates at only one frequency which turns out to be different from the intrinsic oscillation frequency $\varpi$ of the bar, that is evidance in favor of spatial noncommutativity. Since in such a scenario the resonance point must be one of the two possible frequencies [i.e., either at $\left(\varpi+\Lambda_{0}\right)$ or $\left(\varpi-\Lambda_{0}\right)$ ] one can estimate $\Lambda_{0}$ from the observation.

The result in Eq. (65) is consistent with our earlier result in [52] where only spatial noncommutativity has been considered and the result there showed that due to the presence of spatial NC structure the ordinarily expected unique resonance point $\varpi=\Omega$ will split into two evenly spaced resonance points $\Omega=\varpi \pm \Lambda_{\theta}$. Here the only difference is the presence of noncommutativity of the momentum sector $\bar{\theta}$, which splits the expected resonance point as $\Omega=$ $\varpi \pm \Lambda_{0}=\varpi \pm\left(\Lambda_{\theta}-\Lambda_{\bar{\theta}}\right)$. This further shows that if both the spatial and the momentum sector of the phase space have a NC structure, their effects cannot be separately identified, at least by looking at the resonance points. This answers the third question raised in the introduction.

Apart from shifting the ordinarily expected resonance point, the effect of phase-space noncommutativity is also apparant in some terms in the solutions (58), (59) which are proportional to $\frac{f_{0} \Lambda}{4}$ and $\frac{4 f_{0} \Lambda_{\bar{\theta}}}{\varpi}$. We must try to estimate the size of such factors in context of HO system. Curiously, it turns out that these terms have a very different value in connection with the HO system than their corresponding estimates in the free particle context in Sec. III A. To see this we have to consider the experimental setup where quantum mechanical HO's can be realized in GW detectors. We shall briefly elaborate on this after we compute the response of a $\mathrm{HO}$ system to circularly polarized $\mathrm{GW}$ in the next subsection.

Regarding the bound on growth of resonance in the present case the previous arguement given in Sec. III A of a finite observation time still applies. Moreover, even for the monochromatic idealization, which means a periodic GW of infinite duration, the amplitude of the oscillator will keep growing until the damping effect becomes large enough to compensate for the energy absorbed from the GW at which point a stationary regime is reached.

Also note the existence of a smooth commutative limit $(\theta, \bar{\theta}) \rightarrow(0,0)$ i.e., $\left(\Lambda, \Lambda_{\bar{\theta}}\right) \rightarrow(0,0)$ for the solution (58), (59) where the resonance shifts back to the expected frequency $\varpi=\Omega$. We provide the corresponding expressions in the appendix A 2 and demonstrate their consistency.

\section{B. Time evolution under circularly polarized GW}

We shall now solve Eqs. (42), (43) for the circularly polarized GW. We once again substitute the ansatz (23), (24) for the circularly polarized GW (4), (21) in Eqs. (42), (43) to obtain the following set of coupled linear differential equations 
$\dot{A}=-i \varpi E-f_{0} \Omega C-\frac{i 4 \Lambda_{\bar{\theta}}}{\varpi} f_{0} \Omega F-\Lambda_{0} D$,

$\dot{B}=\Omega C-i \varpi F+f_{0} \Omega D-\frac{i 4 \Lambda_{\bar{\theta}}}{\varpi} f_{0} \Omega E+\Lambda_{0} C$,

$\dot{C}=-\Omega B-i \varpi G-f_{0} \Omega A-\frac{i 4 \Lambda_{\bar{\theta}}}{\varpi} f_{0} \Omega H-\Lambda_{0} B$,

$\dot{D}=-i \varpi H+f_{0} \Omega B-\frac{i 4 \Lambda_{\bar{\theta}}}{\varpi} f_{0} \Omega G+\Lambda_{0} A$,

$\dot{E}=-i \varpi A+f_{0} \Omega G+\frac{2 i \Lambda_{\theta}}{\varpi} f_{0} \Omega B-\Lambda_{0} H$,

$\dot{F}=\Omega G-i \varpi B-f_{0} \Omega H+\frac{2 i \Lambda_{\theta}}{\varpi} f_{0} \Omega A+\Lambda_{0} G$,

$\dot{G}=-\Omega F-i \varpi C+f_{0} \Omega E+\frac{2 i \Lambda_{\theta}}{\varpi} f_{0} \Omega D-\Lambda_{0} F$,

$\dot{H}=-i \varpi D-f_{0} \Omega F+\frac{2 i \Lambda_{\theta}}{\varpi} f_{0} \Omega C+\Lambda_{0} E$,

Here $\Lambda_{\bar{\theta}}, \Lambda_{\theta}$ and $\Lambda_{0}$ carry the same meaning as in the earlier subsections. Also note that the constant frequency $\Omega$ defined in (4), (21) is the frequency of the circularly polarized GW. Solving the Eq. (66) to first order in the GW amplitude, with boundary conditions (14), (17) which physically signifies that the GW hits the particle at $t=0$, we get the solutions in terms of the following functions all of which have the dimension of inverse frequency

$V_{1}=\frac{1}{2}\left[\frac{\varpi_{-} \sin \varpi_{+} t+\varpi_{+} \sin \varpi_{-} t}{\varpi_{-} \varpi_{+}}\right]$

$V_{2}=\frac{1}{2}\left[\frac{-2 \varpi+\varpi_{+} \cos \varpi_{-} t+\varpi_{-} \cos \varpi_{+} t}{\varpi_{-} \varpi_{+}}\right]$

$V_{3}=\frac{1}{2}\left[\frac{2 \Lambda_{0}+\varpi_{-} \cos \varpi_{+} t-\varpi_{+} \cos \varpi_{-} t}{\varpi_{-} \varpi_{+}}\right]$

$V_{4}=\frac{1}{2}\left[\frac{\varpi_{+} \sin \varpi_{-} t-\varpi_{-} \sin \varpi_{+} t}{\varpi_{-} \varpi_{+}}\right]$

$V_{5}=\frac{1}{2}\left[\frac{\sin \left(\Omega+\varpi_{-}\right) t}{\left(\Omega+\varpi_{-}\right)}+\frac{\sin \left(\Omega-\varpi_{+}\right) t}{\left(\Omega-\varpi_{+}\right)}\right]$

$V_{6}=\frac{1}{2}\left[\frac{2 \varpi}{\left(\Omega+\varpi_{-}\right)\left(\Omega-\varpi_{+}\right)}-\frac{\cos \left(\Omega+\varpi_{-}\right) t}{\Omega+\varpi_{-}}\right.$

$$
\left.+\frac{\cos \left(\Omega-\varpi_{+}\right) t}{\Omega-\varpi_{+}}\right]
$$

where we have used Eq. (61). Before giving the explicit solutions let us note that the first four functions $V_{1}, V_{2}$, $V_{3}, V_{4}$, despite having a factor $\left(\varpi_{-}\right)^{-1}$ take finite values in the limit $\varpi_{-} \rightarrow 0$. Similarly, $V_{5}$, though have factors like $\left(\Omega \pm \varpi_{ \pm}\right)^{-1}$ also take finite values in the limit $\Omega \rightarrow \varpi_{ \pm}$. Only $V_{6}$ will grow rapidly in the limit $\Omega \rightarrow$ $\left(\Lambda_{0} \pm \varpi\right)$ and this will lead to resonance behavior in the solution. Solutions for $A(t), B(t), C(t), D(t), E(t), F(t)$, $G(t)$ and $H(t)$ are the following:

$$
\begin{aligned}
A(t)= & 1+\varpi V_{2}+\Lambda_{0} V_{3}-f_{0} \Omega V_{5}-i\left(\varpi V_{1}-\Lambda_{0} V_{4}\right. \\
& \left.+\frac{4 \Lambda_{\bar{\theta}} f_{0} \Omega V_{6}}{\varpi}\right) \\
B(t)= & \Omega_{+} V_{5}-f_{0} \Omega V_{3}+\frac{4 \Lambda_{\bar{\theta}} f_{0} \Omega V_{2}}{\varpi}-i\left(\varpi V_{6}+f_{0} \Omega V_{4}\right. \\
& \left.+\frac{4 \Lambda_{\bar{\theta}} f_{0} \Omega V_{1}}{\varpi}\right) \\
C(t)= & -\Omega_{+} V_{6}-f_{0} \Omega V_{1}-\frac{4 \Lambda_{\bar{\theta}} f_{0} \Omega V_{4}}{\varpi}-i\left[\varpi V_{5}+f_{0} \Omega V_{2}\right. \\
& \left.-\frac{4 \Lambda_{\bar{\theta}} f_{0} \Omega V_{3}}{\varpi}\right]
\end{aligned}
$$

$D(t)=\Lambda_{0} V_{1}-\varpi V_{4}+f_{0} \Omega V_{6}-i\left[-\varpi V_{3}-\Lambda_{0} V_{2}\right.$

$$
\left.+\frac{4 \Lambda_{\bar{\theta}} f_{0} \Omega V_{5}}{\varpi}\right]
$$

$$
E(t)=1+\varpi V_{2}+\Lambda_{0} V_{3}+f_{0} \Omega V_{5}-i\left[\varpi V_{1}-\Lambda_{0} V_{4}\right.
$$

$$
\left.-\frac{2 \Lambda_{\theta} f_{0} \Omega V_{6}}{\varpi}\right]
$$

$$
\begin{aligned}
F(t)= & \Omega_{+} V_{5}+f_{0} \Omega V_{3}-\frac{2 \Lambda_{\theta} f_{0} \Omega V_{2}}{\varpi}-i\left[\varpi V_{6}-f_{0} \Omega V_{4}\right. \\
& \left.-\frac{2 \Lambda_{\theta} f_{0} \Omega V_{1}}{\varpi}\right]
\end{aligned}
$$

$$
\begin{aligned}
G(t)= & -\Omega_{+} V_{6}+f_{0} \Omega V_{1}+\frac{2 \Lambda_{\theta} f_{0} \Omega V_{4}}{\varpi}-i\left[\varpi V_{5}-f_{0} \Omega V_{2}\right. \\
& \left.+\frac{2 \Lambda_{\theta} f_{0} \Omega V_{3}}{\varpi}\right]
\end{aligned}
$$

$$
\begin{aligned}
H(t)= & \Lambda_{0} V_{1}-\varpi V_{4}+f_{0} \Omega V_{6}-i\left[-\varpi V_{3}-\Lambda_{0} V_{2}\right. \\
& \left.-\frac{2 \Lambda_{\theta} f_{0} \Omega V_{5}}{\varpi}\right],
\end{aligned}
$$

where we have used Eq. (62). As in the earlier cases, these solutions can be easily cast in the form of positions expectation values at an arbitrary time $\left(\left\langle X_{1}(t)\right\rangle,\left\langle X_{2}(t)\right\rangle\right)$, in terms of the initial position and momentum expectation values $\left(\left\langle X_{1}(0)\right\rangle,\left\langle X_{2}(0)\right\rangle\right)$ and $\left(\left\langle P_{1}(0)\right\rangle,\left\langle P_{2}(0)\right\rangle\right)$ following the algorithm detailed in the discussion in Sec. III A [below (33)]. They read as follows: 


$$
\begin{aligned}
\left\langle X_{1}(t)\right\rangle= & {\left[\left(1+\Lambda_{0} V_{3}+\varpi V_{2}\right)+\left(\Omega+\Lambda_{0}\right)\left(V_{5} \epsilon_{3}-V_{3} \epsilon_{1}\right)\right] X_{1}(0)+\left[\left(\Omega+\Lambda_{0}\right)\left(V_{5} \epsilon_{3}+V_{4} \epsilon_{1}\right)-\Lambda_{0} V_{1}+\varpi V_{4}\right] X_{2}(0) } \\
& +\left[\varpi\left(V_{1}+V_{4} \epsilon_{1}+V_{5} \epsilon_{3}\right)-\Lambda_{0} V_{4}\right] \frac{P_{1}(0)}{m \varpi}+\left[\varpi\left(V_{3}+V_{4} \epsilon_{1}-V_{5} \epsilon_{3}\right)\right] \frac{P_{2}(0)}{m \varpi} \\
& +f_{0} \Omega\left[\left(-V_{6}+V_{3} \epsilon_{3}-V_{1} \epsilon_{1}\right)+\frac{4 \Lambda_{\bar{\theta}}}{\varpi}\left(V_{2} \epsilon_{3}-V_{4} \epsilon_{1}\right)\right] X_{1}(0) \\
& +f_{0} \Omega\left[\left(-V_{6}+V_{3} \epsilon_{1}+V_{1} \epsilon_{3}\right)+\frac{4 \Lambda_{\bar{\theta}}}{\varpi}\left(V_{2} \epsilon_{1}+V_{4} \epsilon_{3}\right)\right] X_{2}(0) \\
& -f_{0} \Omega\left[\left(V_{6}+V_{4} \epsilon_{3}+V_{2} \epsilon_{1}\right)-\frac{2}{\varpi}\left\{2 \Lambda_{\bar{\theta}}\left(V_{6}+V_{1} \epsilon_{3}\right)-\Lambda_{\theta} V_{3} \epsilon_{3}\right\}\right] \frac{P_{1}(0)}{m \varpi} \\
& +f_{0} \Omega\left[\left(-V_{6}-V_{4} \epsilon_{1}+V_{2} \epsilon_{3}\right)+\frac{2}{\varpi}\left\{2 \Lambda_{\bar{\theta}}\left(-V_{5}+V_{1} \epsilon_{1}\right)-\Lambda_{\theta} V_{3} \epsilon_{3}\right\}\right] \frac{P_{2}(0)}{m \varpi} \\
\left\langle X_{2}(t)\right\rangle= & {\left[\left(1+\Lambda_{0} V_{3}+\varpi V_{2}\right)-\left(\Omega+\Lambda_{0}\right)\left(V_{5} \epsilon_{3}-V_{3} \epsilon_{1}\right)\right] X_{2}(0)+\left[\left(\Omega+\Lambda_{0}\right)\left(V_{5} \epsilon_{3}+V_{4} \epsilon_{1}\right)+\Lambda_{0} V_{1}-\varpi V_{4}\right] X_{1}(0) } \\
& +\left[\varpi\left(V_{1}+V_{4} \epsilon_{1}+V_{5} \epsilon_{3}\right)-\Lambda_{0} V_{4}\right] \frac{P_{2}(0)}{m \varpi}+\left[\varpi\left(-V_{3}+V_{4} \epsilon_{1}-V_{5} \epsilon_{3}\right)\right] \frac{P_{1}(0)}{m \varpi} \\
& +f_{0} \Omega\left[\left(-V_{6}+V_{3} \epsilon_{3}-V_{1} \epsilon_{1}\right)-\frac{4 \Lambda_{\bar{\theta}}}{\varpi}\left(V_{2} \epsilon_{3}-V_{4} \epsilon_{1}\right)\right] X_{2}(0) \\
& +f_{0} \Omega\left[\left(V_{6}+V_{3} \epsilon_{1}+V_{1} \epsilon_{3}\right)+\frac{4 \Lambda_{\bar{\theta}}}{\varpi}\left(V_{2} \epsilon_{1}+V_{4} \epsilon_{3}\right)\right] X_{1}(0) \\
& -f_{0} \Omega\left[\left(V_{6}+V_{4} \epsilon_{3}+V_{2} \epsilon_{1}\right)-\frac{2}{\varpi}\left\{2 \Lambda_{\bar{\theta}}\left(V_{6}+V_{1} \epsilon_{3}\right)-\Lambda_{\theta} V_{3} \epsilon_{3}\right\}\right] \frac{P_{2}(0)}{m \varpi} \\
& +f_{0} \Omega\left[\left(-V_{6}-V_{4} \epsilon_{1}+V_{2} \epsilon_{3}\right)+\frac{2}{\varpi}\left\{2 \Lambda_{\bar{\theta}}\left(-V_{5}+V_{1} \epsilon_{1}\right)-\Lambda_{\theta} V_{3} \epsilon_{3}\right\}\right] \frac{P_{1}(0)}{m \varpi} \\
&
\end{aligned}
$$

with polarization vectors following Eq. (36). Once again we look at these solutions critically to point out their salient features. First, note that like in the previous cases, we have expressed the present solutions in a suggestive way where the first four terms represent the oscillatory nature of the $\mathrm{HO}$ system with the $\mathrm{HO}$ intrinsic frequency $\varpi$ shifted by the characteristic NC phase-space frequency $\Lambda_{0}$. These terms are independent of any GW effect (these survive when there is no incoming $\mathrm{GW}$, i.e. $f_{0}=0$ ) whereas the last four terms manifestly show the effect of GW. These latter terms are in general small owing to the extreme smallness of the GW amplitude $f_{0}$. However, due to the rapid growth of the function $V_{6}$, which only appears in these latter set of terms, when the GW frequency approaches the value $\left(\Lambda_{0} \pm \varpi\right)$ these terms will show resonance. This is, of course, the resonance between the GW and the $\mathrm{HO}$ system, shifted by $\Lambda_{0}$. Thus we see that, similar to the linearly polarized case in Sec. IV A, in case of circularly polarized GW also, the $\mathrm{HO}$ will resonate with GW signals. This, along with the result of the previous subsections, answers all three questions posed in the introduction. It tells us that generalizing from the spatial noncommutativity to the phase-space noncommutativity does not alter the basic features of the response of the matter system to linearly or circularly polarized GW and that, as far as the resonance behavior is concerned, the momentum NC scale introduces a new frequency that combines with the corresponding characteristic frequency of the spatial NC scale in such a way that, looking at the resonance point, their effects cannot be distinguished.

As in the earlier cases, the present solution also exhibits the existence of a smooth commutative limit $\left(\Lambda, \Lambda_{\bar{\theta}}\right) \rightarrow(0,0)$. Note that the two resonance points marge to the usual case $\varpi=\Omega$ when we go to the commutative limit. The explicit results in the commutative limit are given in Appendix A 3 and a few interesting observations in that connection are also made there. Similar to the previous section, here also some terms appear with coefficients $\frac{2 f_{0} \Omega \Lambda_{\bar{\theta}}}{\pi}$ and $\frac{2 f_{0} \Omega \Lambda_{\theta}}{\pi}$, which may or may not be of significant size in experimental realization of the quantum mechanical HO in the context of GW detectors. We estimate the size of such terms using the currently available upper bounds of the NC parameters $\theta$ [29] and $\bar{\theta}$ $[12,13]$ in the next subsection.

\section{Realization of quantum mechanical HO in resonant bar detectors: Relative size comparison of various terms}

At present there are mainly two types of GW detectors that are operational, one is the ground-based interferometric detector which operates in a wide frequency spectrum and the other is the resonant bar detector operating in a 
relatively small frequency window. Both these detectors operate in the long wavelength and low velocity regime where our calculation in the present section applies. However whether a quantum mechanical description, as has been carried out in the present paper, applies to the mirrors of an interferometric detector, which is a macroscopic object, is a nontrivial issue that needs further investigation and we wish to address this issue in future. What we would like to point out here is that the quantum mechanical description of $\mathrm{HO}$ system that has been considered in the last two subsections is readily realized in context of the resonant bar detectors pioneered by J.Weber $[67,68]$. These are typical cylindrical Aluminium bars of length $L \sim 3 \mathrm{~m}$ and width $r=30 \mathrm{~cm}$, weighing nearly $M=2 \times 10^{3} \mathrm{Kg}$. For such bar detectors, it can be shown [55] that the fundamental mode of elastic oscillation driven by the passing $\mathrm{GW}$ is identical to a forced harmonic oscillator with effective mass $m_{0}=\frac{M}{2} \sim 10^{3} \mathrm{Kg}$ and frequency $\varpi \sim \frac{\pi v_{s}}{L}=5.6 \mathrm{kHz}$, where $v_{s} \sim 5.4 \mathrm{~km} \mathrm{sec}^{-1}$ is the sound speed within the Aluminium bar at low temperature [69]. Thus, the bar detector, despite being a two-ton macroscopic object, responds to the GW by generating collective elastic oscillation modes that are so tiny in size that a quantum mechanical treatment is necessary and upon quantization they are called the phonon modes. Our quantum mechanical analysis of the $\mathrm{HO}$ system in this section applies to these phonon modes. There are of course higher harmonics present, but we have not taken them into consideration here as they will have much lesser strength.

Using the existing upper bounds on the spatial NC parameter [29] $\theta \sim 10^{-40} \mathrm{~m}^{2}$ the corresponding NC frequency $\Lambda_{\theta}$ for such a $\mathrm{HO}$ mode with KHz-range frequency and $m_{0} \sim 10^{3} \mathrm{Kg}$ effective mass will be

$$
\Lambda_{\theta} \sim\left(\frac{m}{m_{0}}\right)\left(\frac{\varpi}{1 \mathrm{KHz}}\right)^{2}\left(\frac{\theta}{10^{-40} \mathrm{~m}^{2}}\right) \times 0.5 \mathrm{KHz}
$$

i.e., typically in the $\mathrm{KHz}$ range. So it will significantly alter the resonance point. However, for the same $\mathrm{HO}$ mode, the characteristic frequency $\Lambda_{\bar{\theta}}$ set by the momentum NC scale $[12,13]$ will be

$$
\Lambda_{\bar{\theta}} \sim\left(\frac{\bar{\theta}}{2.32 \times 10^{-61} \mathrm{Kg}^{2} \mathrm{~m}^{2} \mathrm{sec}^{-2}}\right)\left(\frac{m_{0}}{m}\right) 0.5 \times 10^{-30} \mathrm{~Hz},
$$

which is way beyond the operating range of the bar detectors and, thus, cannot affect the resonance point. Thus we conclude that though in general the results in the HO-GW interaction case will depend on the composite NC phase-space frequency $\Lambda_{0}$, but for the HO modes in a typical bar detector $\Lambda_{0} \approx \Lambda_{\theta}$.

One should realize that there are a number of static or slowly varying Newtonian forces which affects the detector. However, since the time scale in which these noise sources operate are different than the time scale of $\mathrm{GW}$, there exists a reasonable window of frequency $\left[f_{\min }, f_{\max }\right]$ where GW's effect on the bar oscillation will not be lost in these noises [55]. In the case of resonant bar detectors, the typical detection range is $\sim \mathrm{kHz}$ and using the available estimates of $\theta$ we have already shown in Eq. (71) that the characteristic frequency set by the NC parameter $\Lambda_{0}\left(\approx \Lambda_{\theta}\right)$ turns out to be in the same range. Thus, we expect the resonance at the two said frequencies $\left(\varpi+\Lambda_{0}\right)$ and $\left(\varpi-\Lambda_{0}\right)$ will not be affected by the noise sources.

Let us now consider the dimensionless parameter $\Lambda$ appearing in the solutions (58), (59). In the present case of bar detectors, it takes value of the order of unity:

$$
\Lambda=\frac{m \varpi \theta}{\hbar} \approx 1 \times\left(\frac{m}{m_{0}}\right)\left(\frac{\varpi}{1 \mathrm{KHz}}\right)\left(\frac{\theta}{10^{-40} \mathrm{~m}^{2}}\right) .
$$

So in case of linearly polarized $\mathrm{GW}$, terms in the solutions (58), (59) containing the factor $\frac{f_{0} \Lambda}{4}$ are $\mathcal{O}\left[f_{0}\right]$. They will grow rapidly near the resonance points and affect the system significantly. But terms proportional to $\frac{4 f_{0} \Lambda_{\bar{\theta}}}{\pi}$ will be $\mathcal{O}\left[f_{0} \times 10^{-33}\right]$, so even near resonance they will be much smaller than the other terms.

Since the bar detector operates in the $\mathrm{KHz}$ range, only GWs with similar frequency are of concern here. Thus, $\left(\frac{\Omega}{\varpi}\right) \sim \mathcal{O}[1]$ and terms with coefficients such as $\frac{2 f_{0} \Omega \Lambda_{\theta}}{\varpi}$ and $\frac{2 f_{0} \Omega \Lambda_{\bar{\theta}}}{\varpi}$, appearing in the solutions (69), (70) for circularly polarized $\mathrm{GW}$ are $\mathcal{O}\left[f_{0} \Lambda_{\theta}\right]$ and $\mathcal{O}\left[f_{0} \Lambda_{\bar{\theta}},\right]$, respectively. It is evident from Eqs. (71), (72) and the solutions (69), (70) that while terms with the former coefficient are $\mathcal{O}\left[f_{0}\right]$ and will grow significant near resonance, those with the latter coefficients are $\mathcal{O}\left[f_{0} \times 10^{-33}\right]$ and will remain subdominant in comparison. So in both the cases of linearly and circularly polarized $\mathrm{GW}$, the momentum NC does not affect the result much as far as the bar detectors are concerned. However, in general the response of a $\mathrm{HO}$ system to GW depends on momentum NC parameter and such dependence may become significant for some other realizations of quantum $\mathrm{HO}$ systems as GW detectors.

Before concluding the present section we would like to emphasize that the bar in a given resonant bar detector has one fundamental mode of oscillation intrinsically, given by $\varpi=\frac{\pi v_{s}}{L}$, where $v_{s}$ is the sound speed within the bar material at low temperature and $L$ is the length of the bar [55]. Thus this bar will show resonance with GW at two different frequencies, given by $\left(\varpi+\Lambda_{0}\right)$ and $\left(\varpi-\Lambda_{0}\right)$ if the space indeed has a noncommutative structure. However, since NC structure of space is inherently quantum mechanical in nature, so this phenomenon will only arise when we have quantum mechanical oscillators e.g. the fundamental phonon mode of the bar detector. Thus, in absence of GW although the fundamental phonon mode of the bar will have two oscillation frequencies $\left(\varpi \pm \Lambda_{0}\right)$ if the space is noncommutative, without a GW signal there is nothing to 
resonate with and therefore it will be difficult to observe the effect of such oscillations under ordinary circumstances.

\section{Observational aspects of present day resonant bar detectors and scope for detection of NC structure}

Having theoretically identified the possible effect that a NC structure of the phase space may have on the GW-matter interaction, let us now briefly comment on the detection/ observational aspects in the context of a resonant bar detector of GW where our quantum mechanical analysis of the HO system will have some bearing. The detection of GW in resonant detectors depends on the quantum transitions of the phonon modes from the ground state to higher excited states, which in classical terms amounts to a transfer of energy carried by the GW to the elastic energy of the bar. This will generate a collective coherent motion in the bar which is converted to electrical signals by resonant or parametric transducers and further amplified electrically before detection [55]. In a separate paper [70], we have computed the transition probability for the phonon modes to go to excited states from the ground state by considering the combined effect of spatial noncommutativity and GW as a time-dependent perturbation. The calculation in [70] shows that to search for the NC effect one need not achieve sensitivity goals any higher than what is already needed for a succesful detection of $\mathrm{GW}$ in resonant detectors. However, to date, none of the operational resonant bar detectors in IGEC (International Gravitational Event Collaboration) [38-42] have yet achieved the required quantum limit of sensitivity for a successful detection of $\mathrm{GW}$, though a lot of work is currently being done with the new generation of spherical resonant detectors MiniGrail [43] and Schenberg [44]. To reach the required quantum sensitivity limit and even to overcome it, parametric transducers, which allow quantum squeezing, are being employed in the Schenberg detectors [71,72].

\section{CONCLUDING REMARK}

In the present paper, we have considered how a free particle and harmonic oscillator (HO) in the quantum domain will respond to linearly and circularly polarized gravitational waves $(\mathrm{GW})$ if the given phase space has a noncommutative (NC) structure. The results show resonance behavior in the responses of both free particle and $\mathrm{HO}$ systems to $\mathrm{GW}$ with both kinds of polarizations.

While the HO system with a natural frequency is expected to display resonance with GW of suitable frequency, it is rather curious that a free particle may also show resonance. In our analysis, it turns out that momentum noncommutativity induces an oscillatory behavior in the free particle with a characteristic frequency $\Lambda_{\bar{\theta}}$ and that in turn can resonate with the GW of both polarizations, but spatial noncommutativity does not affect the response in any detectable way. This result, thus, indicates that the possible existence of noncommutativity in the momentum sector of the phase-space algebra can be tested if a GW detector operating in the long-wavelength and low-velocity limit can be realized based on how a quantum-mechanical free particle responds to GW. Any resonance in the response of such a detector may serve as evidence in favor of such noncommutativity. Since such a detection scheme is not yet in place, we can only point out, theoretically, the potential for such a scheme.

In the response of the $\mathrm{HO}$ system (with natural frequency $\varpi$ ) to GW (with frequency $\Omega$ ), we found that both the spatial and the momentum sector of the noncommutative phase-space algebra introduce different characteristic frequencies $\left(\Lambda_{\theta}\right.$ and $\Lambda_{\bar{\theta}}$, respectively) into the system, but these two frequencies combine together to form a composite frequency $\Lambda_{0}=\left(\Lambda_{\theta}-\Lambda_{\bar{\theta}}\right)$ which splits the expected resonance point from $\varpi=\Omega$ to $\varpi=\Omega \pm \Lambda_{0}$. So two equally spaced resonance points instead of a single one at the expected intrinsic frequency of the resonant bar detectors (in which the phonon modes excited by the GW act as the GW-driven $\mathrm{HO}[55]$ and, thus, are the experimental realization of the $\mathrm{HO}$ considered in this paper) may serve as evidence for noncommutativity.

To estimate the size of the NC effects and NC characteristic frequencies, we have used stringent upper bounds on the spatial [29] and momentum [12,13] NC parameters and have taken neutron mass as a reference for a quantum mechanically behaving free particle. We saw that if free neutrons can be subjected to the GW, the corresponding $\Lambda_{\bar{\theta}}$ will be in the Hz-range, whereas the dimensionless parameter $\Lambda$ which comes from the spatial NC sector will be a very small number. This ensures that the spatial noncommutativity does not affect the neutron's response to GW in any measurable way.

On the other hand, the phonon modes which are quantized vibrational modes of the bar detector and behave as the experimental realization of the quantum $\mathrm{HO}$ systems we have considered here, have an effective mass of $\sim 10^{3} \mathrm{Kg}$ in a typical bar detector [55]. With this reference value for the mass of the $\mathrm{HO}$, the dimensionless parameter $\Lambda$ turns out to be approximately unity, spatial NC frequency $\Lambda_{\theta}$ is in the $\mathrm{kHz}$ range and momentum NC frequency $\Lambda_{\bar{\theta}}$ is far below the Hz-range when we use the upper-bound estimates of the corresponding NC parameters. Since the operating frequency for a typical bar detector (which is the same as the intrinsic frequency of the fundamental phonon mode) in also in the $\mathrm{kHz}$ range, one can safely say that in this case the spatial sector of noncommutativity will have a more evident effect than that of the momentum sector. Thus, we see that even though the characteristic frequencies $\Lambda_{\theta}$ and $\Lambda_{\bar{\theta}}$ coming from different sectors of NC algebra appear only as a combination $\Lambda_{0}=\left(\Lambda_{\theta}-\Lambda_{\bar{\theta}}\right)$ in the response of a $\mathrm{HO}$ to $\mathrm{GW}$, it is $\Lambda_{\theta}$ from the spatial NC sector that should make a significant difference in the observation. 
Note that the values for $\left(\Lambda, \Lambda_{\theta}, \Lambda_{\bar{\theta}}\right)$ for the experimental realization of quantum mechanical $\mathrm{HO}$ are in complete contrast with those for the quantum mechanical free particle. We, thus, conclude that the mass (effective or otherwise) of a test body that is subjected to the GW plays a crucial role to determine the relative size of the NC parameter-dependent terms in various solutions and, hence. different NC-dependent response terms may become important in context of different realization of the quantum free particle or harmonic oscillator in various GW detection scenario.

We close our discussion by reminding the readers that the main reason we think of resonant detectors of GW as possible setups where noncommutative quantum harmonic oscillators are realized and where $\mathrm{NC}$ signature can be traced is due to the extreme sensitivity of these instruments owing to the techniques that are used to get rid of the various noise sources such that effectively a length-scale variation $\sim 10^{-20} \mathrm{~m}$ can be detected.

\section{ACKNOWLEDGMENTS}

The authors thank the referee for the comments. A. S. acknowledges the financial support of Department of Science and Technologies (DST) Science and Engineering Research Board (SERB) under Grant No. SR/FTP/PS-208/2012. S. G. acknowledges the financial support of DST SERB under Grant No. YSS/2014/000180.

\section{APPENDIX A: SOLUTIONS IN THE COMMUTATIVE LIMIT}

In this appendix, we give the commutative limits of the solutions obtained for the free particle and $\mathrm{HO}$ systems and show that they satisfy some obvious consistency requirements. We also demonstrate that they reproduce the usual free-particle and HO solutions in their immediately recognizable form when cast in the proper context.

\section{Free particle interacting with circularly polarized GW}

The commutative limits of the solutions (34), (35) are given by

$$
\begin{aligned}
\lim _{\left(\Lambda_{\bar{\theta}}, \Lambda\right) \rightarrow 0}\left\langle X_{1}(t)\right\rangle= & {\left[1+\left(\epsilon_{3}-\epsilon_{1}\right) N_{1}\right] X_{1}(0)+\left[\left(\epsilon_{1}+\epsilon_{3}\right) N_{1}\right] X_{2}(0) } \\
& +\left[t+\frac{\left(N_{2} \epsilon_{3}+N_{3} \epsilon_{1}\right)}{\varpi}+\frac{N_{1}}{\Omega}\left(\epsilon_{1}+\epsilon_{3}\right)\right] \frac{P_{1}(0)}{m}+\left[\frac{\left(N_{2} \epsilon_{1}-N_{3} \epsilon_{3}\right)}{\varpi}+\frac{N_{1}}{\Omega}\left(\epsilon_{1}-\epsilon_{3}\right)\right] \frac{P_{2}(0)}{m} \\
& -f_{0} \Omega\left[\left\{\frac{N_{3}}{\Omega}+\epsilon_{3} t\right\} X_{1}(0)+\left\{\frac{N_{1}}{\Omega}+\epsilon_{1} t\right\} X_{2}(0)\right] \\
& +f_{0} \Omega\left[\left\{-\frac{N_{2}}{\Omega}+\frac{\varpi t^{2}}{2}\left(\epsilon_{3}+\epsilon_{1}\right)\right\} \frac{P_{1}(0)}{m \varpi}+\left\{\frac{N_{3}}{\Omega}+\frac{\varpi t^{2}}{2}\left(\epsilon_{1}-\epsilon_{3}\right)\right\} \frac{P_{2}(0)}{m \varpi}\right] \\
\lim _{\left(\Lambda_{\bar{\theta}}, \Lambda\right) \rightarrow 0}\left\langle X_{2}(t)\right\rangle= & {\left[1-\left(\epsilon_{3}-\epsilon_{1}\right) N_{1}\right] X_{2}(0)+\left[\left(\epsilon_{1}+\epsilon_{3}\right) N_{1}\right] X_{1}(0) } \\
& +\left[t+\frac{\left(N_{2} \epsilon_{3}-N_{3} \epsilon_{1}\right)}{\varpi}-\frac{N_{1}}{\Omega}\left(\epsilon_{1}+\epsilon_{3}\right)\right] \frac{P_{2}(0)}{m}+\left[\frac{\left(N_{2} \epsilon_{1}-N_{3} \epsilon_{3}\right)}{\varpi}+\frac{N_{1}}{\Omega}\left(\epsilon_{1}-\epsilon_{3}\right)\right] \frac{P_{1}(0)}{m} \\
& +f_{0} \Omega\left[\left\{-\frac{N_{3}}{\Omega}+\epsilon_{3} t\right\} X_{2}(0)-\left\{\frac{N_{1}}{\Omega}+\epsilon_{1} t\right\} X_{1}(0)\right] \\
& -f_{0} \Omega\left[\left\{\frac{N_{2}}{\Omega}+\frac{\varpi t^{2}}{2}\left(\epsilon_{3}+\epsilon_{1}\right)\right\} \frac{P_{2}(0)}{m \varpi}-\left\{\frac{N_{3}}{\Omega}-\frac{\varpi t^{2}}{2}\left(\epsilon_{1}-\epsilon_{3}\right)\right\} \frac{P_{1}(0)}{m \varpi}\right]
\end{aligned}
$$

with the dimensionless factors taking the corresponding values

$$
\lim _{\left(\Lambda_{\bar{\theta}}, \Lambda\right) \rightarrow 0} N_{1}=1-\cos \Omega t ; \quad \lim _{\left(\Lambda_{\bar{\theta}}, \Lambda\right) \rightarrow 0} N_{2}=\varpi\left[\frac{\sin \Omega t+\cos \Omega t-1}{\Omega}-t\right] ; \quad \lim _{\left(\Lambda_{\bar{\theta}}, \Lambda\right) \rightarrow 0} N_{3}=\varpi\left[\frac{\sin \Omega t-\cos \Omega t+1}{\Omega}-t\right]
$$

An obvious consistency check for these commutative limit solutions in Eqs. (A1), (A2) (as well as their original NC counterparts in Eqs. (34), (35) is $\left.\left\langle X_{1}(t)\right\rangle\right|_{t=0}=X_{1}(0)$ and $\left.\left\langle X_{2}(t)\right\rangle\right|_{t=0}=X_{2}(0)$ which can be readily verified by noting that at $t=0$ all the dimensionless factors $N_{1}, N_{2}$ and $N_{3}$ in the original set of Eq. (33) and in their commutative limits in Eq. (A3) vanish.

Another interesting observation can be made by considering the commutative limit solutions in Eqs. (A1), (A2) in absence of GW and then taking a further limit $\Omega \rightarrow 0$. Note that due to our special choice of the directional triad $\vec{\varepsilon}$, $\dot{\vec{\varepsilon}}$ and 
$\vec{\varepsilon} \times \dot{\vec{\varepsilon}}$ as axes, in the circularly polarized case, in absence of GW the frequency $\Omega$ refers to some arbitrary rotation frequency of the triad. If this frequency is reduced to a very low value then the solution should assume the familiar form

$$
\left\langle X_{1}(t)\right\rangle=X_{1}(0)+\frac{P_{1}(0)}{m} t ; \quad\left\langle X_{2}(t)\right\rangle=X_{2}(0)+\frac{P_{2}(0)}{m} t
$$

This can also be readily checked in Eqs. (A1), (A2) (with $f_{0}=0$ ) by noting that

$$
\begin{aligned}
\lim _{\left(\Omega, \Lambda_{\bar{\theta}}, \Lambda\right) \rightarrow 0} N_{1} & =\lim _{\Omega \rightarrow 0}(1-\cos \Omega t)=0 \\
\lim _{\left(\Omega, \Lambda_{\bar{\theta}}, \Lambda\right) \rightarrow 0} N_{2} & =\lim _{\Omega \rightarrow 0} \varpi\left[\frac{\sin \Omega t+\cos \Omega t-1}{\Omega}-t\right]=0 \\
\lim _{\left(\Omega, \Lambda_{\bar{\theta}}, \Lambda\right) \rightarrow 0} N_{3} & =\lim _{\Omega \rightarrow 0} \varpi\left[\frac{\sin \Omega t-\cos \Omega t+1}{\Omega}-t\right]=0
\end{aligned}
$$

where in the last two equations l'Hôpital's rule has been employed.

\section{HO interacting with linearly polarized GW}

For the commutative limit of the solution (58), (59) for the HO interacting with linearly polarized GW let us first note the corresponding limiting values that the various frequency parameters take

$$
\begin{gathered}
\lim _{\left(\Lambda_{\bar{\theta}}, \Lambda_{\theta}\right) \rightarrow 0} \varpi_{ \pm}=\varpi(\text { natural frequency of the HO }) ; \lim _{\left(\Lambda_{\bar{\theta}}, \Lambda_{\theta}\right) \rightarrow 0} \Omega_{ \pm}=\Omega(\text { frequency of the GW }) \\
\lim _{\left(\Lambda_{\bar{\theta}}, \Lambda_{\theta}\right) \rightarrow 0} \Delta \varpi_{ \pm}=\Delta \varpi=\varpi-\Omega(\text { difference of the frequencies of the HO and GW })
\end{gathered}
$$

The corresponding commutative limit of these solutions are

$$
\begin{aligned}
\lim _{\left(\Lambda_{\bar{\theta}}, \Lambda_{\theta}, \Lambda\right) \rightarrow 0}\left\langle X_{1}(t)\right\rangle= & \cos \varpi t X_{1}(0)+\sin \varpi t \frac{P_{1}(0)}{m \varpi} \\
& -f_{0} q_{1}(t)\left[\epsilon_{3} X_{1}(0)+\epsilon_{1} X_{2}(0)\right]+f_{0} q_{3}(t)\left[\epsilon_{1} X_{1}(0)+\epsilon_{3} X_{2}(0)\right] \\
& -f_{0} q_{2}(t) \frac{1}{m \varpi}\left[\epsilon_{3} P_{1}(0)+\epsilon_{1} P_{2}(0)\right]-f_{0} q_{4}(t) \frac{1}{m \varpi}\left[\epsilon_{1} P_{1}(0)-\epsilon_{3} P_{2}(0)\right] \\
\lim _{\left(\Lambda_{\bar{\theta}}, \Lambda_{\theta}, \Lambda\right) \rightarrow 0}\left\langle X_{2}(t)\right\rangle= & \cos \varpi t X_{2}(0)+\sin \varpi t \frac{P_{2}(0)}{m \varpi} \\
& +f_{0} q_{1}(t)\left[\epsilon_{3} X_{2}(0)-\epsilon_{1} X_{1}(0)\right]+f_{0} q_{3}(t)\left[-\epsilon_{1} X_{2}(0)+\epsilon_{3} X_{1}(0)\right] \\
& -f_{0} q_{2}(t) \frac{1}{m \varpi}\left[-\epsilon_{3} P_{2}(0)+\epsilon_{1} P_{1}(0)\right]+f_{0} q_{4}(t) \frac{1}{m \varpi}\left[\epsilon_{1} P_{2}(0)+\epsilon_{3} P_{1}(0)\right],
\end{aligned}
$$

with the limiting expressions for the dimensionless functions

$$
\begin{gathered}
\lim _{\left(\Lambda_{\bar{\theta}}, \Lambda_{\theta}\right) \rightarrow 0} q_{1}(t)=-\cos \Delta \varpi t+\frac{\varpi}{(\Delta \varpi)^{2}}\left[-k_{1}(t) \cos \varpi t+k_{2}(t) \sin \varpi t\right] \\
\lim _{\left(\Lambda_{\bar{\theta}}, \Lambda_{\theta}\right) \rightarrow 0} q_{2}(t)=-\sin \Delta \varpi t-\frac{\varpi}{(\Delta \varpi)^{2}}\left[k_{1}(t) \sin \varpi t+k_{2}(t) \cos \varpi t\right] \\
\lim _{\left(\Lambda_{\bar{\theta}}, \Lambda_{\theta}\right) \rightarrow 0} q_{3}(t)=-\frac{\varpi}{(\Delta \varpi)^{2}}\left[k_{1}(t) \sin \varpi t-k_{2}(t) \cos \varpi t\right]+\frac{\varpi^{2}}{(\Delta \varpi)^{2}} \\
\lim _{\left(\Lambda_{\bar{\theta}}, \Lambda_{\theta}\right) \rightarrow 0} q_{4}(t)=-\frac{\varpi}{(\Delta \varpi)^{2}}\left[k_{1}(t) \cos \varpi t+k_{2}(t) \sin \varpi t\right]-\varpi^{2}\left[\frac{\cos \Delta \varpi t}{(\Delta \varpi)^{2}}+\frac{t}{\Delta \varpi}\right]
\end{gathered}
$$


containing resonance at the expected frequency value $\varpi=$ $\Omega$ and functions with the dimension of frequency

$$
\begin{aligned}
\lim _{\left(\Lambda_{\bar{\theta}}, \Lambda_{\theta}\right) \rightarrow 0} k_{1}(t) & =2 \Delta \varpi ; \\
\lim _{\left(\Lambda_{\bar{\theta}}, \Lambda_{\theta}\right) \rightarrow 0} k_{2}(t) & =2 \Omega \sin \Omega t .
\end{aligned}
$$

In the absence of GW $\left(f_{0}=0\right)$, these limiting solutions (A8), (A9) readily become the standard HO solutions. Noting the boundary condition (17), that ensures the arrival of $\mathrm{GW}$ at the $\mathrm{HO}$ site just at $t=0$, one can immidiately see that both the original NC solutions (58), (59) and their commutative limits (A8), (A9) pass the consistency check at $t=0$, just like the free particle solutions in the last section.

\section{HO interacting with circularly polarized GW}

The solutions (69), (70) for the HO interacting with circularly polarized GW go over to their commutative limit smoothly and the limiting solutions are

$$
\begin{aligned}
\lim _{\left(\Lambda_{\bar{\theta}}, \Lambda_{\theta}\right) \rightarrow 0}\left\langle X_{1}(t)\right\rangle= & {\left[\left(1+\varpi V_{2}\right)+\Omega V_{5} \epsilon_{3}\right] X_{1}(0)+\left[\Omega V_{5} \epsilon_{3}\right] X_{2}(0)+\left[\varpi\left(V_{1}+V_{5} \epsilon_{3}\right)\right] \frac{P_{1}(0)}{m \varpi}+\left[-\varpi V_{5} \epsilon_{3}\right] \frac{P_{2}(0)}{m \varpi} } \\
& +f_{0} \Omega\left[\left(-V_{6}-V_{1} \epsilon_{1}\right)\right] X_{1}(0)-f_{0} \Omega\left[\left(-V_{6}+V_{1} \epsilon_{3}\right)\right] X_{2}(0)+f_{0} \Omega\left(V_{6}+V_{2} \epsilon_{1}\right) \frac{P_{1}(0)}{m \varpi} \\
& +f_{0} \Omega\left(-V_{6}+V_{2} \epsilon_{3}\right) \frac{P_{2}(0)}{m \varpi}
\end{aligned}
$$

and

$$
\begin{aligned}
\lim _{\left(\Lambda_{\bar{\theta}}, \Lambda_{\theta}\right) \rightarrow 0}\left\langle X_{2}(t)\right\rangle= & {\left[\left(1+\varpi V_{2}\right)-\Omega V_{5} \epsilon_{3}\right] X_{2}(0)+\left[\Omega V_{5} \epsilon_{3}\right] X_{1}(0)+\left[\varpi\left(V_{1}+V_{5} \epsilon_{3}\right)\right] \frac{P_{2}(0)}{m \varpi}-\left[\varpi V_{5} \epsilon_{3}\right] \frac{P_{1}(0)}{m \varpi} } \\
& -f_{0} \Omega\left[\left(V_{6}+V_{1} \epsilon_{1}\right)\right] X_{2}(0)+f_{0} \Omega\left[\left(V_{6}+V_{1} \epsilon_{3}\right)\right] X_{1}(0)-f_{0} \Omega\left(V_{6}+V_{2} \epsilon_{1}\right) \frac{P_{2}(0)}{m \varpi} \\
& +f_{0} \Omega\left(-V_{6}+V_{2} \epsilon_{3}\right) \frac{P_{1}(0)}{m \varpi}
\end{aligned}
$$

with the functions with dimension of inverse frequency in that limit taking the values

$$
\begin{aligned}
\lim _{\left(\Lambda_{\bar{\theta}}, \Lambda_{\theta}\right) \rightarrow 0} V_{1}=\frac{\sin \varpi t}{\varpi} ; \quad \lim _{\left(\Lambda_{\bar{\theta}}, \Lambda_{\theta}\right) \rightarrow 0} V_{2}=\frac{\cos \varpi t-1}{\varpi} ; \quad \lim _{\left(\Lambda_{\bar{\theta}}, \Lambda_{\theta}\right) \rightarrow 0} V_{3}=\lim _{\left(\Lambda_{\bar{\theta}}, \Lambda\right) \rightarrow 0} V_{4}=0 ; \\
\lim _{\left(\Lambda_{\bar{\theta}}, \Lambda_{\theta}\right) \rightarrow 0} V_{5}=\frac{1}{2}\left[\frac{\sin (\Omega+\varpi) t}{(\Omega+\varpi)}+\frac{\sin (\Omega-\varpi) t}{(\Omega-\varpi)}\right] ; \quad \lim _{\left(\Lambda_{\bar{\theta}}, \Lambda_{\theta}\right) \rightarrow 0} V_{6}=\frac{1}{2}\left[\frac{2 \varpi}{(\Omega+\varpi)(\Omega-\varpi)}-\frac{\cos (\Omega+\varpi) t}{(\Omega+\varpi)}+\frac{\cos (\Omega-\varpi) t}{(\Omega-\varpi)}\right] .
\end{aligned}
$$

Like in the previous two cases we first check the consistency requirement at $t=0$. The boundary condition (17) ensures that the last four terms (showing the effect of GW) in the full NC phase-space solutions (69), (70) and their limits (A15), (A16) given above, drop at $t=0$. We also note that all the functions $V_{1}, V_{2}, V_{3}, V_{4}, V_{5}$ in Eqs. (67) and (A17), which occur in the first four terms in the solutions (69), (70) and (A15), (A16), respectively, vanish at $t=0$. Thus the verification is obvious.

The commutative solutions (A15), (A16) in the absence of GW[73] $\left(f_{0}=0\right)$ represent a HO looked at from a frame with axes rotating with frequency $\Omega$. Thus, in the limit $\Omega \rightarrow 0$, we get back the usual HO solutions [74] $\lim _{\left(\Lambda_{\hat{\theta}}, \Lambda_{\theta}, \Omega\right) \rightarrow 0}\left\langle X_{1}(t)\right\rangle=\cos \varpi t X_{1}(0)+\sin \varpi t \frac{P_{1}(0)}{m \varpi}$

$\lim _{\left(\Lambda_{\bar{\theta}}, \Lambda_{\theta}, \Omega\right) \rightarrow 0}\left\langle X_{2}(t)\right\rangle=\cos \varpi t X_{2}(0)+\sin \varpi t \frac{P_{2}(0)}{m \varpi}$

We note that even though the interaction of $\mathrm{HO}$ with linearly and circularly polarized GW give very different looking general solutions in the NC phase space, in the proper limits both solutions assume identical form, ensuring the consistency of their respective treatments. 
[1] S. Doplicher, K. Fredenhagen, and J. E. Roberts, Phys. Lett. B 331, 39 (1994).

[2] S. Doplicher, K. Fredenhagen, and J. E. Roberts, Commun. Math. Phys. 172, 187 (1995).

[3] D. V. Ahluwalia, Phys. Lett. B 339, 301 (1994).

[4] H. S. Snyder, Phys. Rev. 71, 38 (1947); 72, 68 (1947).

[5] N. Seiberg and E. Witten, J. High Energy Phys. 09 (1999) 032.

[6] See for example, R. J. Szabo, Phys. Rep. 378, 207 (2003) and the references therein.

[7] G. Amelino-Camelia, Living Rev. Relativity 16, 5 (2013).

[8] Since we only consider the NC quantum mechanical phase space, we choose to ignore noncommutativity among the spatial coordinates and time.

[9] J.-z. Zhang, Phys. Rev. Lett. 93, 043002 (2004).

[10] A. E. F. Djemai and H. Smail, Commun. Theor. Phys. 41, 837 (2004).

[11] O. Bertolami, J. G. Rosa, C. Aragao, P. Castorina, and D. Zapall‘a, Phys. Rev. D 72, 025010 (2005).

[12] O. Bertolami, J. G. Rosa, C. M. L. de Aragao, P. Castorina, and D. Zappala, Phys. Rev. D 72, 025010 (2005).

[13] R. Banerjee, B. Dutta Roy, and S. Samanta, Phys. Rev. D 74, 045015 (2006).

[14] Due to the transverse nature of the GW, which we shall choose to be coming along the $z$ direction, it is sufficient to consider the matter part is confined to the $x-y$ plane and, thus, we work with the relevant sector of the phase-space variables and impose NC Heisenberg algebra on the corresponding phase-space operators.

[15] T. P. Singh, S. Gutti, and R. Tibrewala, arXiv:gr-qc/ 0503116.

[16] P. R. Giri and P. Roy, Eur. Phys. J. C 57, 835 (2008).

[17] J. Ben Geloun, S. Gangopadhyay, and F. G. Scholtz, Europhys. Lett. 86, 51001 (2009).

[18] K. Li and S. Dulat, arXiv:0708.3954.

[19] V. P. Nair and A. P. Polychronakos, Phys. Lett. B 505, 267 (2001).

[20] B. Chakraborty, S. Gangopadhyay, and A. Saha, Phys. Rev. D 70, 107707 (2004).

[21] P. M. Ho and H. C. Kho, Phys. Rev. Lett. 88, 151602 (2002).

[22] A. Stern, Phys. Rev. Lett. 100, 061601 (2008).

[23] A. Saha, Eur. Phys. J. C 51, 199 (2007).

[24] A. Saha, Phys. Rev. D 81, 125002 (2010); 89, 025010 (2014).

[25] L. Mezincescu, arXiv:hep-th/0007046.

[26] F. G. Scholtz, B. Chakraborty, S. Gangopadhyay, and A. G. Hazra, Phys. Rev. D 71, 085005 (2005); arXiv:hep-th/ 0502143.

[27] F. G. Scholtz, B. Chakraborty, S. Gangopadhyay, and J. Govaerts, J. Phys. A 38, 9849 (2005).

[28] M. Chaichian, M. M. Sheikh-Jabbari, and A. Tureanu, Phys. Rev. Lett. 86, 2716 (2001).

[29] S. M. Carroll, J. A. Harvey, V. A. Kostelecký, C. D. Lane, and T. Okamoto, Phys. Rev. Lett. 87, 141601 (2001).

[30] A. Abrampvici et al., Science 256, 325 (1992).

[31] B. Caron et al., Classical Quantum Gravity 14, 1461 (1997).

[32] H. Lück et al., Classical Quantum Gravity 14, 1471 (1997).

[33] M. Ando et al., Phys. Rev. Lett. 86, 3950 (2001).

[34] For detailed information on the advanced LIGO project please visit https://www.advancedligo.mit.edu/.

[35] B. P. Abbott et al., Phys. Rev. Lett. 116, 061102 (2016).
[36] B. P. Abbott et al., Phys. Rev. Lett. 116, 241103 (2016).

[37] R. Matzner, in General Relativity and John Archibald Wheeler, Astrophysics and Space Science Library Vol. 367, edited by I. Ciufolini and R. A. Matzner (Springer, New York, 2010).

[38] P. Astone et al., Phys. Rev. D 47, 362 (1993).

[39] E. Mauceli, Z. K. Geng, W. O. Hamilton, W. W. Johnson, S. Merkowitz, A. Morse, B. Price, and N. Solomonson, Phys. Rev. D 54, 1264 (1996).

[40] D. Blair, E. Ivanov, M. Tobar, P. Turner, F. van Kann, and I. Heng, Phys. Rev. Lett. 74, 1908 (1995).

[41] P. Astone et al., Astropart. Phys. 7, 231 (1997).

[42] M. Cerdonio et al., Classical Quantum Gravity 14, 1491 (1997).

[43] A. de Waard, M. Bassan, Y. Benzaim, V. Fafone et al. Classical Quantum Gravity 23, S79 (2006).

[44] O. D. Aguiar, L. A. Andrade, J. J. Barroso et al., Classical Quantum Gravity 23, S239 (2006); C. A. Costa, O. D. Aguiar, N. F. Oliveira, X. Gratens, S. T. de Souza, and S. R. Furtado Classical Quantum Gravity 25, 184002 (2008).

[45] S. Dimopoulos, P. W. Graham, J. M. Hogan, M. A. Kasevich, and S. Rajendran, Phys. Lett. B 678, 37 (2009); arXiv: 0712.1250 .

[46] S. Dimopoulos, P. W. Graham, J. M. Hogan, M. A. Kasevich, and S. Rajendran, Phys. Rev. D 78, 122002 (2008).

[47] D. Gao, P. Ju, B. Zhang, and M. Zhan, Gen. Relativ. Gravit. 43, 2027 (2011).

[48] G. Amelino-Camelia, Nature (London) 398, 216 (1999).

[49] G. Amelino-Camelia, Phys. Rev. D 62, 024015 (2000).

[50] A. D. Speliotopoulos, Phys. Rev. D 51, 1701 (1995).

[51] A. Saha and S. Gangopadhyay, Phys. Lett. B 681, 96 (2009); arXiv:0908.4319.

[52] A. Saha, S. Gangopadhyay, and S. Saha, Phys. Rev. D 83, 025004 (2011); arXiv:1005.3373.

[53] S. Gangopadhyay, A. Saha, and S. Saha, Mod. Phys. Lett. A 28, 1350161 (2013).

[54] S. Gangopadhyay, A. Saha, and S. Saha, Gen. Relativ. Gravit. 47, 28 (2015).

[55] M. Miggiore, Gravitational Wave, Vol I, Theory and Experiments (Oxford University Press, New York, 2008).

[56] It is the same as its proper time to first order in the metric perturbation.

[57] As is usual, latin indices run from 1-3. Also ";" denotes covariant derivatives.

[58] Note that these conditions are not satisfied by the proposed space-borne interferometer eLISA [59] or by the Doppler tracking of spacecraft.

[59] For detailed information on the eLISA project please visit http://www.lisa-science.org.

[60] P. Mukherjee and A. Saha, Phys. Rev. D 74, 027702 (2006) and the references therein.

[61] R. Banerjee, P. Mukherjee, and S. Samanta, Phys. Rev. D 75, 125020 (2007).

[62] In eLISA, there are six free-floating test masses (goldplatinum cubes) which are in free fall and the satellite senses their position in order to measure the distance between the test masses in the different spacecraft.

[63] Actually, owing to the finesse of the LIGO end mirrors, which are about $99 \%$, the light typically travels $\sim 100$ times 
up and down the arm before leaking out, which increases the effective arm length above the physical arm length of $4 \mathrm{~km}$.

[64] See the discussion on how a quantum mechanical treatment of a harmonic oscillator applies for the bar detectors, which are macroscopic objects too, in Sec. IV C.

[65] M. Ando, K. Ishidoshiro, K. Yamamoto, K. Yagi, W. Kokuyama, K. Tsubono, and A. Takamori Phys. Rev. Lett. 105, 161101 (2010).

[66] K. Ishidoshiro1, M. Ando, A. Takamori, H. Takahashi, K. Okada, N. Matsumoto, W. Kokuyama, N. Kanda, Y. Aso, and K. Tsubono, Phys. Rev. Lett. 106, 161101 (2011).

[67] J. Weber, General Relativity and Gravitational Waves (Interscience Publishers, Inc., New York, 1961).

[68] O. D. Aguiar, Res. Astron. Astrophys. 11, 1 (2011) and the reference therein.

[69] Let us also note that at (near) the resonance, the typical reduced wavelength of the $\mathrm{GW} \chi=\frac{c}{\varpi_{0}}$ will be such that
$\frac{L}{\AA}=\frac{\pi v_{s}}{c}=6 \times 10^{-5} \ll 1$, ensuring that the long wavelength, low-velocity limit is satisfied.

[70] A. Saha and S. Gangopadhyay, Classical Quantum Gravity 33, 205006 (2016).

[71] G. L. Pimentel, O. D. Aguiar, J. J. Barroso, and M. E. Tobar, J. Phys. Conf. Ser. 122, 012028 (2008); O. D. Aguiar et al. J. Phys. Conf. Ser. 363, 012003 (2012).

[72] L. A. N. de Paula, E. C. Ferreira, N. C. Carvalho, and O. D. Aguiar, J. Instrum. 10, P03001 (2015).

[73] Through from Eqs. (69), (70), it appears that the $\Omega \rightarrow 0$ limit is enough to get rid of the last four terms, but in the presence of circularly polarized GW, such limit cannot be realized.

[74] Note that the polarization vectors satisfying Eq. (36) in the case of circular polarization become $\left.\epsilon_{1}\right|_{\Omega=0},\left.\epsilon_{3}\right|_{\Omega=0}=(1,0)$ in the $\Omega \rightarrow 0$ limit. 